This is the peer reviewed version of the following article: Z. Anorg. Allg . Chem. 2013, 639, 216-220,

which has been published in final form at: https://doi.org/10.1002/zaac.201300119

ARTICLE

DOI: 10.1002/zaac.200((will be filled in by the editorial staff))

\title{
Synthesis and Single Crystal X-ray Structures of Cationic Zinc $\beta$-Diketiminate Complexes
}

\author{
Christoph Scheiper, ${ }^{[a]}$ Stephan Schulz, ${ }^{*[a]}$ Christoph Wölper, ${ }^{[a]}$ Dieter Bläser, ${ }^{[a]}$ and Joachim \\ Roll $^{[b]}$
}

Dedicated to Prof. H. Nöth on the occasion of his 85th birthday

Keywords: Cations / Zinc / X-ray Analysis

\begin{abstract}
The cationic zinc complex $\left[\mathrm{L}^{1} \mathrm{Zn}\right]\left[\mathrm{Al}\left(\mathrm{OC}\left(\mathrm{CF}_{3}\right)_{3}\right)_{4}\right] \quad(\mathbf{1}) \quad\left(\mathrm{L}^{1}=\right.$ $\left.\left\{\left[\left(2,4,6-\mathrm{Me}_{3}-\mathrm{C}_{6} \mathrm{H}_{2}\right) \mathrm{NC}(\mathrm{Me})\right]_{2} \mathrm{CH}\right\}\right)$ bearing a weakly coordinating aluminate anion was obtained from reaction of $\mathrm{L}^{1} \mathrm{ZnCl}$ with $\mathrm{Li}\left[\mathrm{Al}\left(\mathrm{OC}\left(\mathrm{CF}_{3}\right)_{3}\right)_{4}\right]$. In addition, base-stabilized zinc cations $\left[\mathrm{L}^{1 / 2} \mathrm{Zn}(\text { base })_{2}\right][\mathrm{X}](\mathbf{2}-\mathbf{5})\left(\mathrm{L}^{2}=\left\{\left[\left(2,6-i-\mathrm{Pr}_{2}-\mathrm{C}_{6} \mathrm{H}_{3}\right) \mathrm{NC}(\mathrm{Me})\right]_{2} \mathrm{CH}\right\} ; \mathrm{X}\right.$ $\left.=\left[\mathrm{Al}\left(\mathrm{OC}\left(\mathrm{CF}_{3}\right)_{3}\right)_{4}\right],\left[\mathrm{B}\left(\mathrm{C}_{6} \mathrm{~F}_{5}\right)_{4}\right]\right)$ containing weakly coordinating anions and different Lewis bases (dimethylaminopyridine (dmap), tert-butylpyridine ( $t$-BuPy)) were synthesized and structurally
\end{abstract}

characterized. Moreover, the solid state structures of $\mathrm{L}^{1} \mathrm{ZnMe}$ (6) and the neutral base-stabilized complexes $t$ - $\mathrm{BuPy}-\mathrm{Zn}(\mathrm{Cl}) \mathrm{L}^{1}$ (7) and base- $\mathrm{Zn}(\mathrm{Me}) \mathrm{L}^{2}$ (base $=\operatorname{dmap} \mathbf{8}, t$-BuPy 9) are reported and compared with those of $\mathbf{2}-\mathbf{5}$. In addition, preliminary studies showed that $\mathbf{1}$ is catalytically activity in lactide polymerization at $160^{\circ} \mathrm{C}$.

(C) WILEY-VCH Verlag GmbH \& Co. KGaA, 69451 Weinheim, Germany, 2009) [a] Faculty of Chemistry, University of Duisburg-Essen, Universitätsstr. 5-7, D-45141 Essen

Fax: Int + (0)201 1833830

E-mail: stephan.schulz@uni-due.de

[b] Westfälische Hochschule, University of Applied Sciences, Organometallic Chemistry, August-Schmidt-Ring 10, D45665 Recklinghausen

Supporting information for this article is available on the WWW under http://www.eurjic.org/ or from the author.

\section{Introduction}

The ring opening polymerization of lactide has become a very active research field in the last decade, ${ }^{[1,2]}$ since polylactides have received considerable interest due to their biodegradable properties. ${ }^{[3]}$ Unfortunately, the commercially used catalyst, SnOct $_{2}$, causes problems in the biomedical field due to its toxicity. ${ }^{[2,4]}$ As a consequence, there is an increasing demand in the development of new catalysts, which are non-toxic and of low cost, hence leading to the synthesis of very active zinc, aluminum, magnesium or lanthanide catalysts. ${ }^{[5-8]}$

Organozinc-based catalysts have been investigated for a long time. Early studies of Dittrich and Schulz reported on the use of $\mathrm{ZnMe}_{2}, \mathrm{AlMe}_{3}$ and $\mathrm{SnCl}_{4}$ in the lactide polymerization, ${ }^{[9]}$ whereas Kricheldorf et al. later on introduced more active aluminum alkoxides. ${ }^{[10]}$ However, it was the pioneering work of Coates et al., that clearly set the focus on neutral zinc alkoxide complexes based on monoanionic $\beta$-diketiminato ligands. Complexes of this type were found to show a very high activity and also stereoselectivity in lactide polymerization. ${ }^{[11]}$ Moreover, these complexes were found even to copolymerize $\mathrm{CO}_{2}$ and epoxides. ${ }^{[12]}$ The $\beta$-diketiminato ligands of the type $\left[\mathrm{HC}\{\mathrm{C}(\mathrm{Me}) \mathrm{NR}\}_{2}\right]$ are almost ideal ligands for the synthesis of tailor-made catalysts since their steric demand as well as electronic properties can be precisely controlled by variation of the organic substituents $\mathrm{R}$ bound to the imine centers.
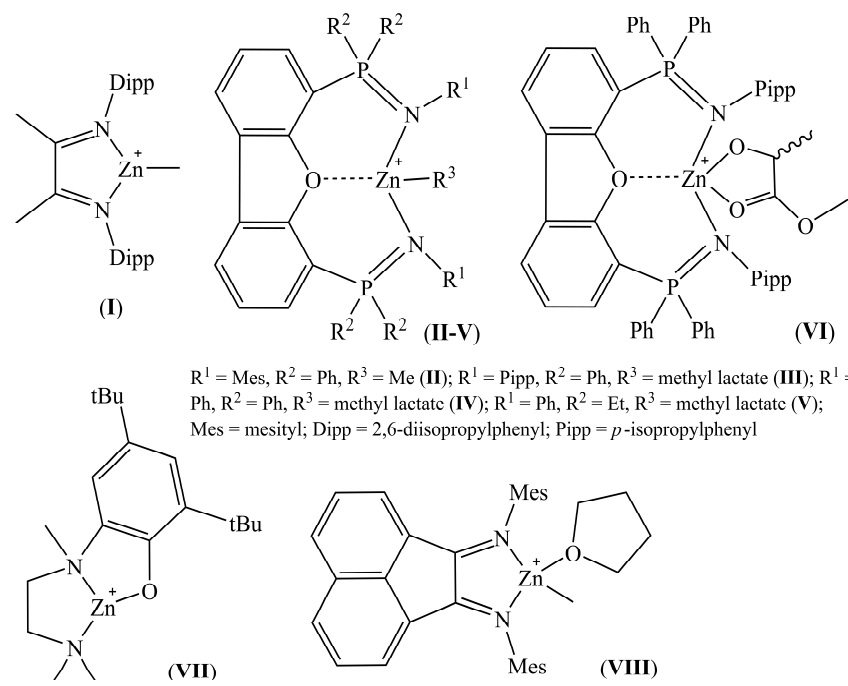

Scheme 1. Catalytically active cationic zinc complexes.

Based on the experimental finding, that zwitterionic tris(pyrazolyl)borate complexes are active polymerization catalysts, ${ }^{[13]}$ Hayes et al. suggested, that the activity of metal complexes in the lactide polymerization can be increased by use of more electropositive metals. ${ }^{[14]}$ Aside from changing the metal atom, the acidity of a cationic metal complex is also higher compared to its neutral form, hence promoting facile coordination of lactide. Cationic metal complexes have been shown to be effective catalysts in the ring- 
opening polymerization of lactones, ${ }^{[15]}$ whereas lactide polymerization has been studied to a lesser extent. ${ }^{[16]}$

Structurally characterized cationic complexes containing coordinative saturated zinc atoms are well known, ${ }^{[17]}$ whereas the number of structurally characterized threecoordinated cationic zinc complexes was limited for a long time to Bochmann cationic zinc diazadiene complexes. ${ }^{[15 \mathrm{~b}, \mathrm{f}]}$ Recently, Hayes et al. synthesized three-coordinated cationic zinc complexes bearing weakly coordinating anions based on mono(phosphinimine)dibenzofuran as well as on bis(phosphinimine)dibenzofuran pincer-type ligands (type II). ${ }^{[18,19]}$ Unfortunately, these cationic alkyl zinc complexes of the type $[\mathrm{LZnR}]^{+}(\mathrm{R}=\mathrm{Me}, \mathrm{Ph})$ were found to be almost inactive for the polymerization of lactide. However, the steric demand of the organic ligand bound to the imine center as well as the alkyl group bound to the $\mathrm{Zn}$ center were found to play a crucial role on the catalytic activity of the complex. For instance, the cationic complex VI containing the sterically less demanding Pipp-substituent $($ Pipp $=p$-isopropyl phenyl) and a zinc-lactate moiety was found to polymerize 200 equiv. of lactide within $50 \mathrm{~min}$ at ambient temperature. ${ }^{[20]}$ In contrast, the sterically less demanding Ph-substituted complex IV is unexpectedly less active than VI, most likely due to electronic effects. ${ }^{[21]}$ The phenyl group on the $\mathrm{N}$-aryl rings reduces the electron donating capacity of the ligand, whereby the zinc center in IV is more electropositive and reduces its polymerization activity. The exchange of the bulky phenyl ligands at the phosphorus atoms by sterically less demanding ethyl groups yielded $\mathbf{V},{ }^{[22]}$ which showed an increased catalytic activity. 200 equiv. of lactide were polymerized within $20 \mathrm{~min}$ at ambient temperature, clearly demonstrating the crucial role of steric and electronic effects on the resulting catalytic activity. Very recently, cationic zinc complexes containing both three- and four-coordinated zinc atoms were successfully used in polymerization reactions. ${ }^{[16 f, g, 19-23]}$ Three-coordinated cationic complexes of type VII are active in lactide polymerization and trimethylene carbonate polymerization. ${ }^{[16 \mathrm{~g}]}$ In addition, the base-stabilized complex VIII was found to polymerize $\varepsilon$-caprolactone without the need of additional initiators such as alcohols. ${ }^{[23]}$

Herein, we report on the synthesis of base-free and basestabilized cationic zinc complexes based on $\beta$-diketiminato ligands. The latter were also structurally characterized by single crystal X-ray diffraction. Furthermore, the catalytic activity of $\left[\mathrm{L}^{1} \mathrm{Zn}\right]\left[\mathrm{Al}\left\{\mathrm{OC}\left(\mathrm{CF}_{3}\right)_{3}\right\}_{4}\right]$ (1) in the ring opening polymerization of lactide was investigated.

\section{Results and Discussion}

$\mathrm{L}^{1} \mathrm{ZnCl}\left(\mathrm{L}^{1}=\left\{\left[\left(2,4,6-\mathrm{Me}_{3}-\mathrm{C}_{6} \mathrm{H}_{2}\right) \mathrm{NC}(\mathrm{Me})\right]_{2} \mathrm{CH}\right\}^{[24]}\right)$ reacts

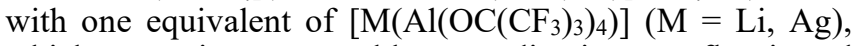
which contains a weakly coordinating perfluorinated alkoxyaluminate anion, ${ }^{[25,26]}$ with subsequent formation of $\left[\mathrm{L}^{1} \mathrm{Zn}\right]\left[\mathrm{Al}\left\{\mathrm{OC}\left(\mathrm{CF}_{3}\right)_{3}\right\}_{4}\right]$ (1) in high yield. 1 is soluble in $\mathrm{CH}_{2} \mathrm{Cl}_{2}$ upon warming to $40{ }^{\circ} \mathrm{C}$ and precipitates slowly as colorless crystalline solid upon cooling to ambient temperature. ${ }^{1} \mathrm{H}$ and ${ }^{13} \mathrm{C}$ NMR spectra of 1 show two sets of resonances due to the Mesnacnac ligand, most likely indicating the coordination of one mesityl group to the electrophilic $\mathrm{Zn}$ cation as was observed in the solid state structure of the neutral amidinate complex $[t$ $\left.\mathrm{BuC}(\mathrm{NDipp})_{2} \mathrm{ZnMe}\right] .^{[27]}$ Unfortunately, numerous attempts to grow suitable crystals for a single crystal X-ray diffraction study failed, to date.

Since the solubility of the cationic zinc $\beta$-diketiminate complexes $\left[\mathrm{L}^{1 / 2} \mathrm{Zn}\right]^{+}[\text {Anion }]^{-}$was expected to be increased by coordination of strong Lewis bases to the electrophilic zinc center, we reacted $\mathrm{L}^{1} \mathrm{ZnCl}$ and $\mathrm{L}^{2} \mathrm{ZnMe}$ with $\mathrm{M}\left[\mathrm{Al}\left\{\mathrm{OC}\left(\mathrm{CF}_{3}\right)_{3}\right\}_{4}\right](\mathrm{M}=\mathrm{Li}, \mathrm{Ag})$ and $\mathrm{Ph}_{3} \mathrm{C}[\mathrm{X}]\left[\mathrm{X}^{1}=\right.$ $\left[\mathrm{Al}\left\{\mathrm{OC}\left(\mathrm{CF}_{3}\right)_{3}\right\}_{4}\right], \mathrm{X}^{2}=\left[\mathrm{B}\left(\mathrm{C}_{6} \mathrm{~F}_{5}\right)_{4}\right]$, respectively, in the presence of two equivalents of either 4dimethylaminopyridine (dmap) or $t$-BuPy. The reaction progress on the reaction with $\mathrm{Ph}_{3} \mathrm{C}[\mathrm{X}]$, which is given by the formation of $\mathrm{Ph}_{3} \mathrm{CMe}$, can be in situ monitored by ${ }^{1} \mathrm{H} \mathrm{NMR}$ spectroscopy.

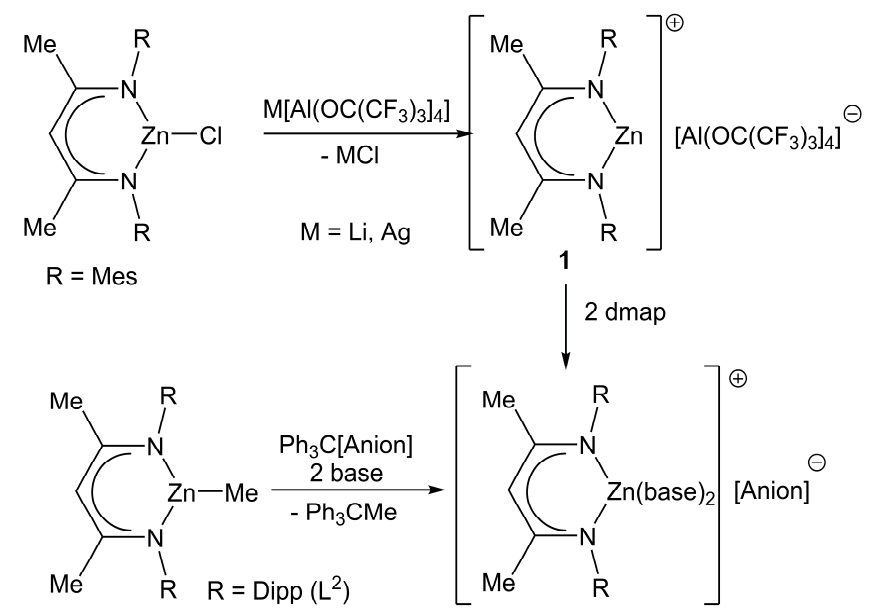

Anion $=\mathrm{Al}\left(\mathrm{OC}\left(\mathrm{CF}_{3}\right)_{3}\right]_{4} \mathrm{R}=$ Mes, base $=$ dmap 2, $\mathrm{R}=$ Dipp, $t-$ BuPy 3 Anion $=\mathrm{B}\left(\mathrm{C}_{6} \mathrm{~F}_{5}\right)_{4}, \mathrm{R}=$ Dipp, base $=$ dmap 4, $t$-BuPy 5

Scheme 2. Synthetic routes to cationic $\beta$-diketiminate zinc complexes.

Base-stabilized $\left[\mathrm{L}^{1} \mathrm{Zn}(\mathrm{dmap})_{2}\right]\left[\mathrm{Al}\left\{\mathrm{OC}\left(\mathrm{CF}_{3}\right)_{3}\right\}_{4}\right]$ (2) and $\left[\mathrm{L}^{2} \mathrm{Zn}(\text { base })_{2}\right]\left[\mathrm{X}^{1 / 2}\right]\left(\mathrm{L}^{2}=\left\{\left[\left(2,6-i-\mathrm{Pr}_{2}-\mathrm{C}_{6} \mathrm{H}_{3}\right) \mathrm{NC}(\mathrm{Me})\right]_{2} \mathrm{CH}\right\}\right.$ (Dippnacnac); $\mathrm{X}^{1}=\left[\mathrm{Al}\left\{\mathrm{OC}\left(\mathrm{CF}_{3}\right)_{3}\right\}_{4}\right]$, base $=t$-BuPy $(3), \mathrm{X}^{2}$ $=\left[\mathrm{B}\left(\mathrm{C}_{6} \mathrm{~F}_{5}\right)_{4}\right]$, base $=\operatorname{dmap}(4), t$-BuPy $\left.(5)\right)$ were obtained in high yields $(>80 \%)$. The resonances of the Lewis bases (dmap, $t$-BuPy) are shifted toward higher $(\mathrm{CH})$ and lower field $\left(\mathrm{CH}_{3}\right)$ compared to those of the "free" bases. Moreover, the relative intensity of the resonances due to the anionic $\beta$ diketiminato group and the Lewis bases (dmap, $t$-BuPy) of 1:2 indicates the formation of stable bis-adducts in solution. Complexes $\mathbf{2}-\mathbf{5}$, which are soluble in organic solvents such as $\mathrm{CH}_{2} \mathrm{Cl}_{2}$, toluene or benzene, do not show any sign of decomposition over a period of several days.

2 - 5 were structurally characterized by single crystal Xray diffraction. The complexes, which show almost identical structural parameters (Table 1), each consist of a twofold base-stabilized zinc cation and the respective anion, which shows no interactions to the cationic zinc center. The zinc centers in the cations adopt a tetrahedral coordination sphere as was previously reported for cationic zinc complexes. ${ }^{[26,32,16 \mathrm{~h}]}$ Analogous findings were observed for the corresponding neutral base-stabilized complexes $t$-BuPy$\mathrm{Zn}(\mathrm{Cl}) \mathrm{L}^{1}(7)$ and base- $\mathrm{Zn}(\mathrm{Me}) \mathrm{L}^{2}$ (base = dmap 8, $t$-BuPy 9), which have been prepared by equimolar reaction of $\mathrm{L}^{1} \mathrm{ZnCl}$ and $\mathrm{L}^{2} \mathrm{ZnMe}$ with the corresponding Lewis base and structurally characterized in order to compare their structural 
parameters with those of $\mathbf{2}-\mathbf{5}$. In contrast, $\mathrm{L}^{1} \mathrm{ZnMe} \mathbf{6}$ exhibits a threefold-coordinated $\mathrm{Zn}$ center. ${ }^{[29 \mathrm{c}]}$

The bonding parameters of the $\beta$-diketiminate ligand frameworks as well as the $\mathrm{Zn}-\mathrm{N}$ bond distances of the sixmembered $\mathrm{ZnN}_{2} \mathrm{C}_{3}$ rings in $\mathbf{2}-\mathbf{5}$ are within the typical range as reported by us and others. ${ }^{[29]}$ The $\mathrm{C}-\mathrm{N}$ and $\mathrm{C}-\mathrm{C}$ bond lengths within the $\beta$-diketiminate backbones are almost identical compared to those of the neutral base stabilized complexes $t$-BuPy-Zn(Cl)L $\mathrm{L}^{1}(7)$, dmap- $\mathrm{Zn}(\mathrm{Me}) \mathrm{L}^{2}(8)$, and $t$ BuPy-Zn(Me)L ${ }^{2}$ (9) (Table 2).

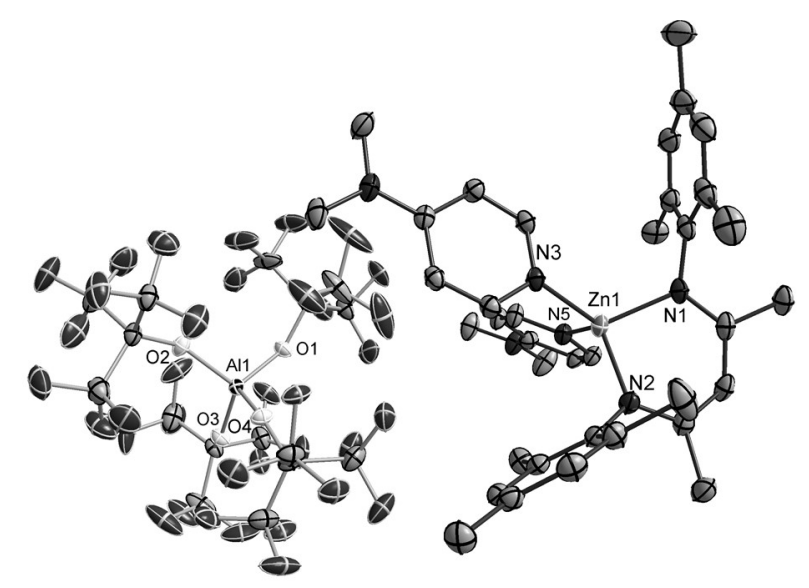

Figure 1. Crystal structure of $\left[\mathrm{L}^{1} \mathrm{Zn}(\mathrm{dmap})_{2}\right]\left[\mathrm{Al}\left\{\mathrm{OC}\left(\mathrm{CF}_{3}\right)_{3}\right\}_{4}\right]$ (2). All hydrogen atoms and alternative site of the disorder have been omitted for clarity. Ellipsoids are drawn at $50 \%$ probability level.

Table 1. Central bond lengths $(\AA)$ and bond angles $\left(^{\circ}\right)$ of

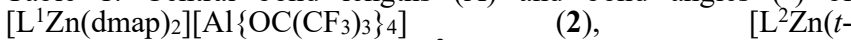

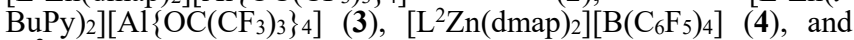
$\left[\mathrm{L}^{2} \mathrm{Zn}(t-\mathrm{BuPy})_{2}\right]\left[\mathrm{B}\left(\mathrm{C}_{6} \mathrm{~F}_{5}\right)_{4}\right](\mathbf{5})$.

\begin{tabular}{lllll}
\hline & $\mathbf{2}$ & $\mathbf{3}$ & $\mathbf{4}$ & $\mathbf{5}$ \\
\hline Zn-N1/2 & $1.9595(18)$ & $1.955(3)$ & $1.9723(8)$ & $1.9562(13)$ \\
& $1.9638(17)$ & $1.956(4)$ & $1.9682(18)$ & $1.9602(14)$ \\
Zn-Namine & $1.9947(17)$ & $2.060(4)$ & $1.9935(18)$ & $2.0257(14)$ \\
& $2.0488(18)$ & $2.020(4)$ & $2.0540(19)$ & $2.0417(14)$ \\
N1-C1 & $1.328(3)$ & $1.331(5)$ & $1.333(3)$ & $1.332(2)$ \\
N2-C3 & $1.327(3)$ & $1.321(5)$ & $1.318(3)$ & $1.328(2)$ \\
C1-C2 & $1.404(3)$ & $1.401(6)$ & $1.400(3)$ & $1.408(2)$ \\
C2-C3 & $1.404(3)$ & $1.408(6)$ & $1.422(3)$ & $1.409(2)$ \\
N1-Zn1- & $97.50(7)$ & $99.19(14)$ & $98.75(7)$ & $99.19(6)$ \\
N2 & & & & \\
Zn1-N1- & $119.12(15)$ & $118.5(3)$ & $116.46(14)$ & $117.83(11)$ \\
C1 & & & & \\
Zn1-N2- & $118.74(15)$ & $119.2(3)$ & $117.42(15)$ & $119.01(11)$ \\
C3 & & & & \\
C1-C2-C3 & $128.7(2)$ & $129.7(4)$ & $129.6(2)$ & $129.43(15)$ \\
\hline
\end{tabular}

Table 2. Central bond lengths $(\AA)$ and bond angles $\left(^{\circ}\right)$ of $\left[\mathrm{L}^{1} \mathrm{ZnMe}\right]$ (6) and base-stabilized, neutral zinc complexes $t$-BuPy-Zn(Cl) $\mathrm{L}^{1}$ (7) as well as base-Zn(Me) $\mathrm{L}^{2}($ base $=\operatorname{dmap} \mathbf{8}, t$-BuPy 9).

\begin{tabular}{lllll}
\hline & $\mathbf{6}$ & $\mathbf{7}$ & $\mathbf{8}$ & $\mathbf{9}$ \\
\hline Zn-N1/2 & $1.946(2)$ & $1.9842(12)$ & $2.023(2)$ & $2.0423(11)$ \\
& $1.9596(16)$ & $1.9803(12)$ & $2.028(2)$ & $2.0251(10)$ \\
Zn-Namine & - & $2.0699(12)$ & $2.097(2)$ & $2.1464(11)$ \\
Zn-Cl & - & $2.2074(4)$ & - & -
\end{tabular}

\begin{tabular}{lllll}
$\mathrm{Zn}-\mathrm{C}$ & $1.946(2)$ & - & $1.995(3)$ & $1.9825(14)$ \\
$\mathrm{N} 1-\mathrm{C} 1$ & $1.327(2)$ & $1.3251(18)$ & $1.331(3)$ & $1.3178(15)$ \\
$\mathrm{N} 2-\mathrm{C} 3$ & $1.336(3)$ & $1.3243(18)$ & $1.327(3)$ & $1.3279(16)$ \\
$\mathrm{C} 1-\mathrm{C} 2$ & $1.408(3)$ & $1.410(2)$ & $1.410(3)$ & $1.4130(17)$ \\
$\mathrm{C} 2-\mathrm{C} 3$ & $1.399(3)$ & $1.407(2)$ & $1.403(4)$ & $1.4051(18)$ \\
$\mathrm{N} 1-\mathrm{Zn} 1-$ & $96.02(7)$ & $97.48(5)$ & $94.62(9)$ & $93.90(4)$ \\
$\mathrm{N} 2$ & & & & \\
Zn1-N1- & $123.42(13)$ & $120.27(9)$ & $122.30(18)$ & $122.85(8)$ \\
$\mathrm{C} 1$ & & & & \\
$\mathrm{Zn} 1-\mathrm{N} 2-$ & $122.69(13)$ & $120.61(9)$ & $122.07(17)$ & $121.60(9)$ \\
$\mathrm{C} 3$ & & & & \\
$\mathrm{C} 1-\mathrm{C} 2-\mathrm{C} 3$ & $128.62(18)$ & $129.12(13)$ & $130.0(2)$ & $128.98(11)$ \\
\hline
\end{tabular}

In contrast, the neutral complexes 7 - 9 show slightly elongated $\mathrm{Zn}-\mathrm{N}$ bond lengths compared to those of the cationic complexes $\mathbf{2}$ - 5, most likely resulting from the increased electrophilicity of the cationic zinc centers and due to higher ionic bonding contributions, as well as slightly larger $\mathrm{Zn}-\mathrm{N}-\mathrm{C}$ bond angles. $\mathrm{L}^{1} \mathrm{ZnMe}$ (6), which only contains a threefold-coordinated $\mathrm{Zn}$ center, shows almost identical bonding parameters compared to the cationic complex 2. Moreover, the $\mathrm{Zn}-\mathrm{N}$ bond lengths compare very well with the values obtained from DFT calculations for $\mathrm{L}^{1} \mathrm{ZnMe}(2.0025,2.0018)^{[29 \mathrm{c}]}$ and $\mathrm{L}^{2} \mathrm{ZnMe}$ (1.9480(18), 1.9429(18) A), respectively. ${ }^{[2]}$ The $\mathrm{Zn}-\mathrm{N}_{\text {amine }}$ bond lengths to the coordinating Lewis bases are significantly longer as was expected (Table 1).
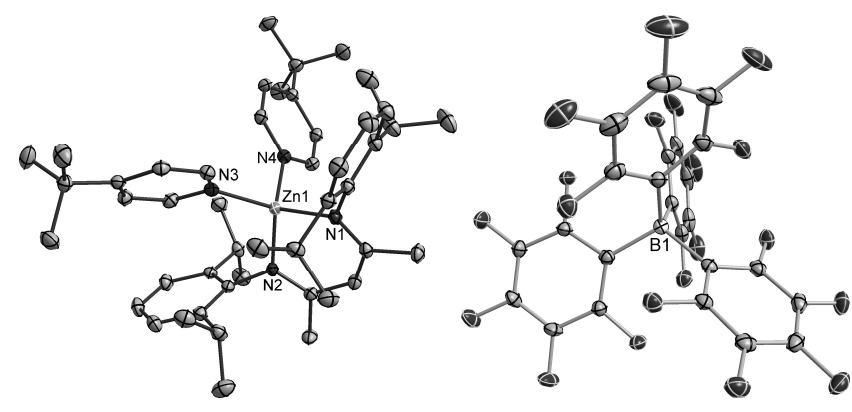

Figure 2. Crystal structure of $\left[\mathrm{L}^{2} \mathrm{Zn}(t-\mathrm{BuPy})_{2}\right]\left[\mathrm{B}\left(\mathrm{C}_{6} \mathrm{~F}_{5}\right)_{4}\right]$ (5). All hydrogen atoms as well as the solvent molecule have been omitted for clarity. Ellipsoids are drawn at $50 \%$ probability level. The relative orientation of the ions does not resemble their orientation in the asymetric unit.

Preliminary polymerization studies. Initial studies demonstrated the capability of complex 1 to serve as catalyst in ring opening polymerization (ROP) of lactide.

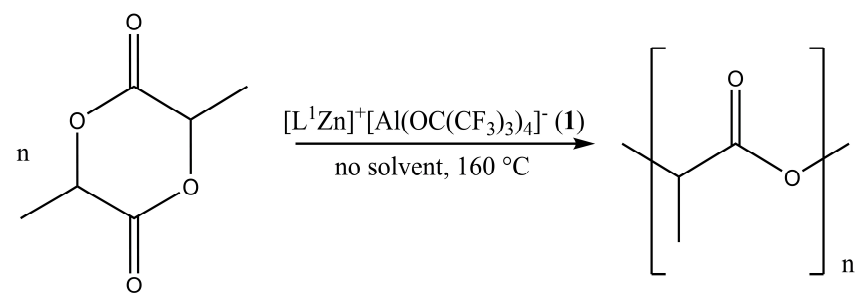

Scheme 3. Ring opening polymerization of lactide by use of bulk $\mathbf{1}$.

Since $\mathbf{1}$ is not soluble in organic solvents at room temperature, its catalytic activity could not be investigated at ambient temperature. However, $\mathbf{1}$ is active in bulk 
polymerization at $160{ }^{\circ} \mathrm{C}$, converting 200 equivalents of lactide within $8 \mathrm{~min}$ into polylactide. The molecular weight $\left(\mathrm{M}_{\mathrm{W}} ; 1.2 \cdot 10^{5} \mathrm{~g} / \mathrm{mol}\right)$ and the polydispersity index (PDI; 1.73) of the resulting polylactide was determined by gel permeation chromatography (GPC) in hexafluoroisopropanol (HFIP) with $0.05 \mathrm{M}$ potassiumtrifluoracetate (KTFAc) at $23{ }^{\circ} \mathrm{C}$ (flow $1 \mathrm{~mL} / \mathrm{min}$ ) using a refractive index detector $(\mathrm{RI})$.

\section{Conclusions}

The base-free cationic zinc complex

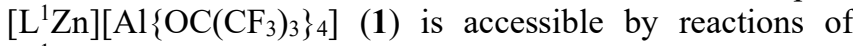
$\left[\mathrm{L}^{1} \mathrm{ZnCl}\right]$ with $\mathrm{M}\left[\mathrm{Al}\left\{\mathrm{OC}\left(\mathrm{CF}_{3}\right)_{3}\right\}_{4}\right](\mathrm{M}=\mathrm{Li}, \mathrm{Ag})$. In contrast, reactions of $\left[\mathrm{L}^{2} \mathrm{ZnMe}\right]$ with $\mathrm{Ph}_{3} \mathrm{CX}^{1 / 2}$ in the presence of two equivalents of a Lewis base yielded base-stabilized complexes $\left[\mathrm{L}^{1} \mathrm{Zn}(\text { dmap })_{2}\right]\left[\mathrm{Al}\left\{\mathrm{OC}\left(\mathrm{CF}_{3}\right)_{3}\right\}_{4}\right] \quad$ (2), $\quad\left[\mathrm{L}^{2} \mathrm{Zn}(t\right.$ -

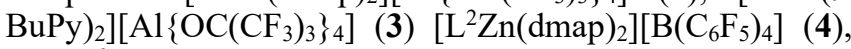
and $\left[\mathrm{L}^{2} \mathrm{Zn}(t-\mathrm{BuPy})_{2}\right]\left[\mathrm{B}\left(\mathrm{C}_{6} \mathrm{~F}_{5}\right)_{4}\right](5)$, respectively, which were fully characterized including single crystal $\mathrm{X}$-ray analyses. $\left[\mathrm{L}^{1} \mathrm{Zn}\right]\left[\mathrm{Al}\left\{\mathrm{OC}\left(\mathrm{CF}_{3}\right)_{3}\right\}_{4}\right] \mathbf{1}$ is an active lactide polymerization catalyst in its bulk form at $160{ }^{\circ} \mathrm{C}$. Detailed studies on the catalytic activity of $\mathbf{1}$ as well as of base-stabilized complexes $\mathbf{2}$ - 5 in ROP of lactide are currently under investigation.

\section{Experimental Section}

General: All manipulations involving air-sensitive materials were performed using standard vacuum techniques and inert gas atmosphere (argon, $<0.3 \mathrm{ppm} \mathrm{O}_{2}$ ) or in a glove box (MBraun Unilab V2.0). Solvents were carefully dried $\left(\mathrm{CH}_{2} \mathrm{Cl}_{2}\right)$ with $\mathrm{CaH}_{2}$, degassed and stored in teflon-sealed glass vessel over appropriate drying agents (molecular sieve). $\mathrm{L}^{1} \mathrm{ZnR}(\mathrm{R}=\mathrm{Me}(\mathbf{6}), \mathrm{Cl}),{ }^{[29 \mathrm{c}]}$ $\mathrm{L}^{2} \mathrm{ZnR}(\mathrm{R}=\mathrm{Me}, \mathrm{Cl}),{ }^{[24,30]} \mathrm{M}\left[\mathrm{Al}\left\{\mathrm{OC}\left(\mathrm{CF}_{3}\right)_{3}\right\} 4\right](\mathrm{M}=\mathrm{Li}, \mathrm{Ag}),{ }^{[25]}$ $\mathrm{Ph}_{3} \mathrm{C}\left[\mathrm{Al}\left\{\mathrm{OC}\left(\mathrm{CF}_{3}\right)_{3}\right\} 4\right],{ }^{[31]} \mathrm{Li}\left[\mathrm{B}\left(\mathrm{C}_{6} \mathrm{~F}_{5}\right)_{4}\right],{ }^{[32]}$ and $\mathrm{Ph}_{3} \mathrm{C}\left[\mathrm{B}\left(\mathrm{C}_{6} \mathrm{~F}_{5}\right)_{4}\right]^{[32]}$ were synthesized according to literature methods. ${ }^{1} \mathrm{H},{ }^{13} \mathrm{C}$ and ${ }^{19} \mathrm{~F}$ NMR spectra were collected with a Bruker Advance 300 spectrometer and are referenced to internal $\mathrm{CD}_{2} \mathrm{Cl}_{2}\left({ }^{1} \mathrm{H}: \delta=5.36\right.$, $\left.{ }^{13} \mathrm{C}: \delta=53.4\right)$ and external $\mathrm{CCl}_{3} \mathrm{~F}\left({ }^{19} \mathrm{~F}: \delta=0.31\right)$. Elemental analyses were performed at the Labor für Elementaranalyse of the University of Duisburg-Essen. GPC data, the average molar mass $\left(\mathrm{M}_{\mathrm{n}}\right)$ and molar mass distribution $\left(\mathrm{M}_{\mathrm{w}} / \mathrm{M}_{\mathrm{n}}\right)$ were obtained on a gel permeation chromatography (GPC) in hexafluoroisopropanol (HFIP) with $0.05 \mathrm{M}$ potassiumtrifluoracetate (KTFAc) at $23{ }^{\circ} \mathrm{C}$ (flow rate $1 \mathrm{~mL} / \mathrm{min}$ ). Three column (PSS-PFG 1000; 300; $100 \AA$, $7 \mu \mathrm{m})$ were used in combination with a refractive index and diode array detector and calibrated with polymethylmethacrylate. The polymer samples were dissolved in $\operatorname{HFIP}(3.4 \mathrm{mg} / \mathrm{mL})$ at $23^{\circ} \mathrm{C}$.

[L $\left.\mathbf{L}^{1} \mathbf{Z n}\right]\left[\mathbf{A l}\left\{\mathbf{O C}\left(\mathbf{C F}_{3}\right)_{3}\right\}_{4}\right] \quad(\mathbf{1}): \mathrm{L}^{1} \mathrm{ZnCl}(0.43 \mathrm{~g}, 1.0 \mathrm{mmol})$ and $\mathrm{Li}\left(\mathrm{Al}\left(\mathrm{OC}\left(\mathrm{CF}_{3}\right)_{3}\right)_{4}(0.97 \mathrm{~g}, 1.0 \mathrm{mmol})\right.$ were dissolved in $50 \mathrm{ml}$ of $\mathrm{CH}_{2} \mathrm{Cl}_{2}$ and heated to $60{ }^{\circ} \mathrm{C}$ for $1 \mathrm{~h}$. The resulting suspension was hot filtered and the solvent of the filtrate evacuated under vacuum to give 1 as a colorless solid $(1.11 \mathrm{~g}, 81 \%)$. Elemental analysis calcd (\%) for $\mathrm{C}_{39} \mathrm{H}_{29} \mathrm{~N}_{2} \mathrm{ZnAlO}_{4} \mathrm{~F}_{36}$ : C 34.3, H 2.1, N 2.1; found: $\mathrm{C}$ 37.5, H 2.8, N 2.0. ${ }^{1} \mathrm{H}$ NMR ( $\left.300 \mathrm{MHz}, \mathrm{CD}_{2} \mathrm{Cl}_{2}, 2{ }^{\circ} \mathrm{C}\right): \delta=1.54$ $\left(\mathrm{s}, 3 \mathrm{H}, \beta-\mathrm{CCH}_{3}{ }^{1}\right), 1.76\left(\mathrm{~s}, 6 \mathrm{H}, o-\mathrm{CH}_{3}{ }^{1}\right), 1.86\left(\mathrm{~s}, 6 \mathrm{H}, o-\mathrm{CH}_{3}{ }^{2}\right), 2.06$ (s, $\left.3 \mathrm{H}, \beta-\mathrm{CCH}_{3}{ }^{2}\right), 2.46\left(\mathrm{~s}, 3 \mathrm{H}, p-\mathrm{CH}_{3}{ }^{1}\right), 2.47\left(\mathrm{~s}, 3 \mathrm{H}, p-\mathrm{CH}_{3}{ }^{2}\right), 3.98$ (s, $1 \mathrm{H}, \gamma-\mathrm{CH}), 6.82\left(\mathrm{~s}, 2 \mathrm{H}, m-\mathrm{H}^{1}\right), 6.91\left(\mathrm{~s}, 2 \mathrm{H}, m-\mathrm{H}^{2}\right) ;{ }^{13} \mathrm{C} \mathrm{NMR}$ $\left(75 \mathrm{MHz}, \mathrm{CD}_{2} \mathrm{Cl}_{2}, 25{ }^{\circ} \mathrm{C}\right): \delta=17.4\left(o-\mathrm{CH}_{3}{ }^{1}\right), 17.6\left(o-\mathrm{CH}_{3}{ }^{2}\right), 20.7$ $\left(\beta-\mathrm{CCH}_{3}{ }^{1}\right), 20.9\left(\beta-\mathrm{CCH}_{3}{ }^{2}\right), 22.9\left(p-\mathrm{CH}_{3}{ }^{1}\right), 24.9\left(p-\mathrm{CH}_{3}{ }^{2}\right), 95.1(\gamma-$ C), $121.2\left(\mathrm{q}, \mathrm{J}_{\mathrm{C}-\mathrm{F}}=292.3 \mathrm{~Hz}, \mathrm{CF}_{3}\right), 127.6\left(m-\mathrm{C}^{1}\right), 129.3\left(m-\mathrm{C}^{2}\right)$, $130.3\left(o-C^{1}\right), 131.5\left(o-C^{2}\right), 133.9\left(p-C^{1}\right), 137.3\left(p-C^{2}\right), 138.7\left(i-C^{1}\right)$,
$143.0\left(i-\mathrm{C}^{2}\right), 169.9\left(\beta-C^{1} \mathrm{CH}_{3}\right), 183.7\left(\beta-C^{2} \mathrm{CH}_{3}\right) .{ }^{19} \mathrm{~F}$ NMR (282 $\left.\mathrm{MHz}, \mathrm{CDCl}_{3}, 25^{\circ} \mathrm{C}\right) \delta=-75.4$.

[[ $\left.\mathbf{L}^{\mathbf{1}} \mathbf{Z n}(\mathbf{d m a p})_{2}\right]\left[\mathbf{A l}\left\{\mathbf{O C}\left(\mathbf{C F}_{3}\right)_{3}\right\}_{4}\right]$ (2): $\mathrm{L}^{1} \mathrm{ZnCl}(0.22 \mathrm{~g}, 0.5 \mathrm{mmol})$ was dissolved in $40 \mathrm{~mL}$ of $\mathrm{CH}_{2} \mathrm{Cl}_{2} . \mathrm{Ag}\left(\mathrm{Al}\left(\mathrm{OC}\left(\mathrm{CF}_{3}\right)_{3}\right)_{4}(0.54 \mathrm{~g}, 0.5\right.$ $\mathrm{mmol})$ and dmap (0.12 g, $1.0 \mathrm{mmol})$ were added and the resulting mixture was stirred for $18 \mathrm{~h}$ at room temperature. The solution was filtered, concentrated to $5 \mathrm{~mL}$ and stored at $-30{ }^{\circ} \mathrm{C}$. Colorless crystals of 2 were formed within $24 \mathrm{~h}(0.72 \mathrm{~g}, 89 \%)$. Elemental analysis calcd (\%) for $\mathrm{C}_{53} \mathrm{H}_{49} \mathrm{~N}_{6} \mathrm{ZnAlO}_{4} \mathrm{~F}_{36}$ : C 39.5, H 3.1, N 5.2; found: C 39.6, $\mathrm{H} \mathrm{3.3,} \mathrm{N} \mathrm{5.5.}{ }^{1} \mathrm{H} \mathrm{NMR}\left(300 \mathrm{MHz}, \mathrm{CD}_{2} \mathrm{Cl}_{2}, 25^{\circ} \mathrm{C}\right)$ : $\delta=1.80\left(\mathrm{~s}, 6 \mathrm{H}, \beta-\mathrm{CCH}_{3}\right), 1.88\left(\mathrm{~s}, 12 \mathrm{H}, o-\mathrm{CH}_{3}\right), 2.31(\mathrm{~s}, 6 \mathrm{H}, p-$ $\left.\mathrm{CH}_{3}\right), 3.05\left(\mathrm{~s}, 12 \mathrm{H}, \mathrm{N}\left(\mathrm{CH}_{3}\right)_{2}\right), 5.01(\mathrm{~s}, 1 \mathrm{H}, \gamma-\mathrm{CH}), 6.40\left(\mathrm{~d},{ }^{3} \mathrm{~J}_{\mathrm{HH}}=\right.$ $\left.7.2 \mathrm{~Hz}, 4 \mathrm{H}, \mathrm{C}(3)-\mathrm{H}_{\mathrm{dmap}}\right) 6.82(\mathrm{~s}, 4 \mathrm{H}, m-\mathrm{H}), 7.11\left(\mathrm{~d},{ }^{3} \mathrm{~J}_{\mathrm{HH}}=7.2 \mathrm{~Hz}\right.$, $\left.4 \mathrm{H}, \mathrm{C}(2)-\mathrm{H}_{\mathrm{dmap}}\right) ;{ }^{13} \mathrm{C} \mathrm{NMR}\left(75 \mathrm{MHz}, \mathrm{CD}_{2} \mathrm{Cl}_{2}, 25{ }^{\circ} \mathrm{C}\right) \delta=17.9(o-$ $\left.\mathrm{CH}_{3}\right), 20.4\left(\beta-\mathrm{CCH}_{3}\right), 20.6\left(p-\mathrm{CH}_{3}\right), 39.0\left(\mathrm{~N}_{\left.\left(\mathrm{CH}_{3}\right)_{2}\right),} 93.8(\gamma-\mathrm{C})\right.$, $107.1\left(\mathrm{C}(3)_{\mathrm{dmap}}\right), 121.2$ (q, J-F $\left.=293.3 \mathrm{~Hz}, \mathrm{CF}_{3}\right), 129.3(m-\mathrm{C})$, $131.6(o-\mathrm{C}), 134.4(p-\mathrm{C}), 144.2$ (i-C), 147.3 (C(2) dmap), 155.5 (C(4)dmap), $168.6\left(\beta-\mathrm{CCH}_{3}\right) .{ }^{19} \mathrm{~F}$ NMR $\left(282 \mathrm{MHz}, \mathrm{CD}_{2} \mathrm{Cl}_{2}, 25^{\circ} \mathrm{C}\right) \delta$ $=-75.7$

[L' $\left.\mathbf{L}^{2} \mathbf{Z n}(\boldsymbol{t}-\mathbf{B u P y})_{2}\right]\left[\mathbf{A l}\left\{\mathbf{O C}\left(\mathbf{C F}_{3}\right)_{3}\right\}_{4}\right]$ (3): $\mathrm{L}^{2} \mathrm{ZnMe}(0.25 \mathrm{~g}, 0.5 \mathrm{mmol})$ was dissolved in $40 \mathrm{~mL}$ of $\mathrm{CH}_{2} \mathrm{Cl}_{2} . \mathrm{Ph}_{3} \mathrm{C}\left(\mathrm{Al}\left(\mathrm{OC}_{(\mathrm{CF}}\right)_{3}\right)_{4}(0.61 \mathrm{~g}$, $0.5 \mathrm{mmol})$ and $t$-BuPy $(0.14 \mathrm{~g}, 1.0 \mathrm{mmol})$ were added and the resulting mixture was stirred for $18 \mathrm{~h}$ at room temperature. The solvent was removed at reduced pressure and the colorless solid extracted with $n$-pentane $(20 \mathrm{~mL}) .3$ was obtained after storage at $30{ }^{\circ} \mathrm{C}$ as colorless crystals $(0.68 \mathrm{~g}, 79 \%)$. Elemental analysis calcd (\%) for $\mathrm{C}_{63} \mathrm{H}_{67} \mathrm{~N}_{4} \mathrm{ZnAlO}_{4} \mathrm{~F}_{36}$ : C 44.0, H 3.9, $\mathrm{N} 3.3$; found: C 45.6, $\mathrm{H} 4.0, \mathrm{~N} 3.0 .{ }^{1} \mathrm{H}$ NMR $\left(300 \mathrm{MHz}, \mathrm{CD}_{2} \mathrm{Cl}_{2}, 25{ }^{\circ} \mathrm{C}\right): \delta=0.52(\mathrm{~d}$, $\left.{ }^{3} \mathrm{~J}_{\mathrm{HH}}=6.8 \mathrm{~Hz}, 12 \mathrm{H}, \mathrm{CH}\left(\mathrm{CH}_{3}{ }^{1}\right)_{2}\right), 1.14\left(\mathrm{~d},{ }^{3} \mathrm{~J}_{\mathrm{HH}}=6.8 \mathrm{~Hz}, 12 \mathrm{H}\right.$, $\left.\mathrm{CH}\left(\mathrm{CH}_{3}{ }^{2}\right)_{2}\right), 1.33\left(\mathrm{~s}, 18 \mathrm{H}, \mathrm{C}\left(\mathrm{CH}_{3}\right)_{3}\right), 1.95\left(\mathrm{~s}, 6 \mathrm{H}, \beta-\mathrm{CCH}_{3}\right), 2.90$ (sept, $\left.{ }^{3} \mathrm{~J}_{\mathrm{HH}}=6.8 \mathrm{~Hz}, 4 \mathrm{H}, \mathrm{CH}\left(\mathrm{CH}_{3}\right)_{2}\right), 5.15(\mathrm{~s}, 1 \mathrm{H}, \gamma-\mathrm{CH}), 7.19-7.28$ $(6 \mathrm{H}, m / p-\mathrm{H}), 7.47\left(\mathrm{~d},{ }^{3} \mathrm{~J}_{\mathrm{HH}}=6.4 \mathrm{~Hz}, 4 \mathrm{H}, \mathrm{C}(3)-\mathrm{HPy}\right), 7.52\left(\mathrm{~d},{ }^{3} \mathrm{~J}_{\mathrm{HH}}=\right.$ $\left.6.4 \mathrm{~Hz}, 4 \mathrm{H}, \mathrm{C}(2)-\mathrm{H}_{\mathrm{Py}}\right) .{ }^{13} \mathrm{C} \mathrm{NMR}\left(75 \mathrm{MHz}, \mathrm{CD}_{2} \mathrm{Cl}_{2}, 25^{\circ} \mathrm{C}\right) \delta=$ $23.2 \quad\left(\mathrm{CH}\left(\mathrm{CH}_{3}{ }^{1}\right)_{2}\right), \quad 23.5 \quad\left(\beta-\mathrm{CCH}_{3}\right), \quad 24.0 \quad\left(\mathrm{CH}\left(\mathrm{CH}_{3}{ }^{2}\right)_{2}\right), \quad 28.3$

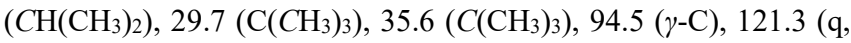
$\left.\mathrm{J}_{\mathrm{C}-\mathrm{F}}=293.3 \mathrm{~Hz}, \mathrm{CF}_{3}\right), 123.6(\mathrm{C}(3) \mathrm{Py}), 124.4(m-\mathrm{C}), 126.5(p-\mathrm{C})$, $142.2(o-\mathrm{C}), 143.0(i-\mathrm{C}), 148.2(\mathrm{C}(2) \mathrm{Py}), 167.4(\mathrm{C}(4) \mathrm{Py}), 170.5(\beta-$ $\left.\mathrm{CCH}_{3}\right) .{ }^{19} \mathrm{~F}$ NMR $\left(282 \mathrm{MHz}, \mathrm{CD}_{2} \mathrm{Cl}_{2}, 25^{\circ} \mathrm{C}\right) \delta=-75.7$.

[ $\left.\mathbf{L}^{2} \mathbf{Z n}(\mathbf{d m a p})_{2}\right]\left[\mathbf{B}\left(\mathbf{C}_{6} \mathbf{F}_{5}\right)_{4}\right]$ (4): $\mathrm{L}^{2} \mathrm{ZnMe}(0.25 \mathrm{~g}, 0.5 \mathrm{mmol})$ was dissolved in $40 \mathrm{~mL}$ of $\mathrm{CH}_{2} \mathrm{Cl}_{2}$. $\mathrm{Ph}_{3} \mathrm{C}\left(\mathrm{B}\left(\mathrm{C}_{6} \mathrm{~F}_{5}\right)_{4}\right)(0.46 \mathrm{~g}, 0.5 \mathrm{mmol})$ and two equivalents of dmap $(0.12 \mathrm{~g}, 1.0 \mathrm{mmol})$ were added and the resulting mixture was stirred for $18 \mathrm{~h}$ at $60^{\circ} \mathrm{C}$. The solvent was removed at reduced pressure and the resulting light green powder extracted with $n$-pentane. 4 was obtained as colorless crystalline solid after storage at $-30{ }^{\circ} \mathrm{C}$ for $24 \mathrm{~h}(0.61 \mathrm{~g}, 86 \%)$. Elemental analysis calcd (\%) for $\mathrm{C}_{67} \mathrm{H}_{61} \mathrm{~N}_{6} \mathrm{ZnBF}_{20}$ : C 57.2, $\mathrm{H} 4.4, \mathrm{~N} 6.0$; found: $\mathrm{C}$ 61.3, $\mathrm{H} 4.5, \mathrm{~N} 4.9 .{ }^{1} \mathrm{H} \mathrm{NMR}\left(300 \mathrm{MHz}, \mathrm{CD}_{2} \mathrm{Cl}_{2}, 25^{\circ} \mathrm{C}\right)$ : $\delta=0.67\left(\mathrm{~d},{ }^{3} \mathrm{~J}_{\mathrm{HH}}=6.78 \mathrm{~Hz}, 12 \mathrm{H}, \mathrm{CH}\left(\mathrm{CH}_{3}{ }^{1}\right)_{2}\right), 1.15\left(\mathrm{~d},{ }^{3} \mathrm{~J}_{\mathrm{HH}}=6.8\right.$ $\left.\mathrm{Hz}, 12 \mathrm{H}, \mathrm{CH}\left(\mathrm{CH}_{3}{ }^{2}\right)_{2}\right), 1.89\left(\mathrm{~s}, 6 \mathrm{H}, \beta-\mathrm{CCH}_{3}\right), 3.04(\mathrm{~s}, 12 \mathrm{H}$, $\left.\mathrm{N}\left(\mathrm{CH}_{3}\right)_{2}\right), 3.04\left(\mathrm{sept},{ }^{3} \mathrm{~J}_{\mathrm{HH}}=6.8 \mathrm{~Hz}, 4 \mathrm{H}, \mathrm{CH}\left(\mathrm{CH}_{3}\right)_{2}\right), 5.04(\mathrm{~s}, 1 \mathrm{H}, \gamma-$ $\mathrm{CH}), 6.40\left(\mathrm{~d},{ }^{3} \mathrm{~J}_{\mathrm{HH}}=6.80 \mathrm{~Hz}, 4 \mathrm{H}, \mathrm{C}(3)-\mathrm{H}_{\mathrm{dmap}}\right), 7.10\left(\mathrm{~d},{ }^{3} \mathrm{~J}_{\mathrm{HH}}=6.80\right.$ $\left.\mathrm{Hz}, 4 \mathrm{H}, \mathrm{C}(2)-\mathrm{H}_{\mathrm{dmap}}\right), 7.20-7.28(6 \mathrm{H}, m / p-\mathrm{H}) ;{ }^{13} \mathrm{C} \mathrm{NMR}(75 \mathrm{MHz}$, $\left.\mathrm{CD}_{2} \mathrm{Cl}_{2}, 25{ }^{\circ} \mathrm{C}\right) \delta=23.4\left(\mathrm{CH}\left(\mathrm{CH}_{3}{ }^{1}\right)_{2}\right), 23.5\left(\beta-\mathrm{CCH}_{3}\right), 24.0$ $\left(\mathrm{CH}\left(\mathrm{CH}_{3}{ }^{2}\right)_{2}\right), 28.3\left(\mathrm{CH}\left(\mathrm{CH}_{3}\right)_{2}\right), 39.0\left(\mathrm{~N}\left(\mathrm{CH}_{3}\right)_{2}\right), 94.0(\gamma-\mathrm{C}), 107.2$ (C(3)dmap), 124.1 ( $m$-C), 129.0 ( $p$-C), $134.6\left(i-\mathrm{C}_{6} \mathrm{~F}_{5}\right), 137.9$ (m$\left.\mathrm{C}_{6} \mathrm{~F}_{5}\right), 142.4(o-\mathrm{C}), 143.7(i-\mathrm{C}), 146.5\left(p-\mathrm{C}_{6} \mathrm{~F}_{5}\right), 147.5$ (C(2)dmap), $149.7\left(o-\mathrm{C}_{6} \mathrm{~F}_{5}\right), 155.4(\mathrm{C}(4) \mathrm{dmap}), 169.7\left(\beta-\mathrm{CCH}_{3}\right) .{ }^{19} \mathrm{~F}$ NMR $(282$ $\left.\mathrm{MHz}, \mathrm{CD}_{2} \mathrm{Cl}_{2}, 25^{\circ} \mathrm{C}\right) \delta=-167.6,-163.8,-133.1$.

[ $\left.\mathbf{L}^{2} \mathbf{Z n}(\boldsymbol{t}-\mathbf{B u P y})_{2}\right]\left[\mathbf{B}\left(\mathbf{C}_{6} \mathbf{F}_{5}\right)_{4}\right]$ (5): $\mathrm{L}^{2} \mathrm{ZnMe}(0.25 \mathrm{~g}, 0.5 \mathrm{mmol})$ was dissolved in $40 \mathrm{~mL}$ of $\mathrm{CH}_{2} \mathrm{Cl}_{2} . \mathrm{Ph}_{3} \mathrm{C}\left(\mathrm{B}\left(\mathrm{C}_{6} \mathrm{~F}_{5}\right)_{4}\right)(0.46 \mathrm{~g}, 0.5 \mathrm{mmol})$ and $t$-BuPy $(0.14 \mathrm{~g}, 1.0 \mathrm{mmol})$ were added and the resulting mixture was stirred for $18 \mathrm{~h}$ at room temperature. The solvent was removed at reduced pressure and the colorless solid extracted with 
$n$-pentane $(20 \mathrm{~mL}) .5$ was obtained as colorless crystalline solid after storage at $-30{ }^{\circ} \mathrm{C}$ for $24 \mathrm{~h}(0.67 \mathrm{~g}, 94 \%)$. Elemental analysis calcd (\%) for $\mathrm{C}_{71} \mathrm{H}_{67} \mathrm{~N}_{4} \mathrm{ZnBF}_{20} * \mathrm{CH}_{2} \mathrm{Cl}_{2}$ : C 57.3, H 4.5, N 3.7; found: C 56.9, $\mathrm{H} 4.6, \mathrm{~N} 3.5 .{ }^{1} \mathrm{H}$ NMR $\left(300 \mathrm{MHz}, \mathrm{CD}_{2} \mathrm{Cl}_{2}, 25{ }^{\circ} \mathrm{C}\right)$ : $\delta=0.52\left(\mathrm{~d},{ }^{3} \mathrm{~J}_{\mathrm{HH}}=6.8 \mathrm{~Hz}, 12 \mathrm{H}, \mathrm{CH}\left(\mathrm{CH}_{3}{ }^{1}\right)_{2}\right), 1.14\left(\mathrm{~d},{ }^{3} \mathrm{~J}_{\mathrm{HH}}=6.8 \mathrm{~Hz}\right.$ $\left.12 \mathrm{H}, \mathrm{CH}\left(\mathrm{CH}_{3}{ }^{2}\right)_{2}\right), 1.33\left(\mathrm{~s}, 18 \mathrm{H}, \mathrm{C}\left(\mathrm{CH}_{3}\right)_{3}\right), 1.95\left(\mathrm{~s}, 6 \mathrm{H}, \beta-\mathrm{CCH}_{3}\right)$, $2.90\left(\mathrm{sept},{ }^{3} \mathrm{~J}_{\mathrm{HH}}=6.8 \mathrm{~Hz}, 4 \mathrm{H}, \mathrm{CH}\left(\mathrm{CH}_{3}\right)_{2}\right), 5.15(\mathrm{~s}, 1 \mathrm{H}, \gamma-\mathrm{CH}), 7.19$ $7.28(6 \mathrm{H}, m / p-\mathrm{H}), 7.47\left(\mathrm{~d},{ }^{3} \mathrm{~J}_{\mathrm{HH}}=6.4 \mathrm{~Hz}, 4 \mathrm{H}, \mathrm{C}(3)-\mathrm{H}_{\mathrm{Py}}\right), 7.52(\mathrm{~d}$, $\left.{ }^{3} \mathrm{~J}_{\mathrm{HH}}=6.4 \mathrm{~Hz}, 4 \mathrm{H}, \mathrm{C}(2)-\mathrm{HPy}_{\mathrm{Py}}\right) \cdot{ }^{13} \mathrm{C} \mathrm{NMR}\left(75 \mathrm{MHz}, \mathrm{CD}_{2} \mathrm{Cl}_{2}, 25{ }^{\circ} \mathrm{C}\right)$ $\delta=23.2\left(\mathrm{CH}\left(\mathrm{CH}_{3}{ }^{1}\right)_{2}\right), 23.5\left(\beta-\mathrm{CCH}_{3}\right), 24.0\left(\mathrm{CH}\left(\mathrm{CH}_{3}{ }^{2}\right)_{2}\right), 28.3$ $\left(\mathrm{CH}\left(\mathrm{CH}_{3}\right)_{2}\right), 29.7\left(\mathrm{C}\left(\mathrm{CH}_{3}\right)_{3}\right), 35.6\left(\mathrm{C}_{\left.\left(\mathrm{CH}_{3}\right)_{3}\right),} 94.5(\gamma-\mathrm{C}), 123.6\right.$ $(\mathrm{C}(3) \mathrm{Py}), 124.4(m-\mathrm{C}), 126.5(p-\mathrm{C}), 134.6\left(i-\mathrm{C}_{6} \mathrm{~F}_{5}\right), 137.9\left(m-\mathrm{C}_{6} \mathrm{~F}_{5}\right)$, $142.2(o-\mathrm{C}), 143.0(i-\mathrm{C}), 146.5\left(p-\mathrm{C}_{6} \mathrm{~F}_{5}\right), 148.2(\mathrm{C}(2) \mathrm{Py}), 149.7(o-$ $\left.\mathrm{C}_{6} \mathrm{~F}_{5}\right), 167.4(\mathrm{C}(4) \mathrm{Py}), 170.5\left(\beta-\mathrm{CCH}_{3}\right) .{ }^{19} \mathrm{~F} \mathrm{NMR}(282 \mathrm{MHz}$, $\left.\mathrm{CD}_{2} \mathrm{Cl}_{2}, 25^{\circ} \mathrm{C}\right) \delta=-167.6,-163.8,-133.1$.

$\boldsymbol{t}$-BuPy-Zn(Cl)L ${ }^{1}$ (7): $\mathrm{L}^{1} \mathrm{ZnCl}(0.43 \mathrm{~g}, 1.0 \mathrm{mmol})$ and t-BuPy $(0.14$ $\mathrm{g}, 1.0 \mathrm{mmol}$ ) were dissolved in $50 \mathrm{ml}$ of THF and the resulting mixture was stirred for $18 \mathrm{~h}$ at room temperature. The solution was concentrated to $5 \mathrm{~mL}$ and stored at $-30{ }^{\circ} \mathrm{C}$. Colorless crystals of 7 were formed within $24 \mathrm{~h}(0.52 \mathrm{~g}, 92 \%)$. Elemental analysis calcd (\%) for $\mathrm{C}_{32} \mathrm{H}_{42} \mathrm{ClN}_{3} \mathrm{Zn}$ : C 67.5, H 7.4, N 7.4; found: C 66.3, H 7.3, $\mathrm{N} 7.1 .{ }^{1} \mathrm{H}$ NMR $\left(300 \mathrm{MHz}, \mathrm{CD}_{2} \mathrm{Cl}_{2},-80{ }^{\circ} \mathrm{C}\right): \delta=1.23(\mathrm{~s}, 9 \mathrm{H}$, $\left.\mathrm{C}\left(\mathrm{CH}_{3}\right)_{3}\right), 1.41\left(\mathrm{~s}, 6 \mathrm{H}, o-\mathrm{CH}_{3}{ }^{1}\right), 1.54\left(\mathrm{~s}, 6 \mathrm{H}, o-\mathrm{CH}_{3}^{2}\right), 2.09(\mathrm{~s}, 6 \mathrm{H}$, $\beta$ - $\left.\mathrm{CCH}_{3}\right), 2.17$ (s, 6H, $p$ - $\left.\mathrm{CH}_{3}\right), 4.76$ (s, 1H, $\left.\gamma-\mathrm{CH}\right), 6.70$ (s, 2H, m$\left.\mathrm{H}^{1}\right), 6.83\left(\mathrm{~s}, 2 \mathrm{H}, m-\mathrm{H}^{2}\right), 7.39\left(\mathrm{~d},{ }^{3} \mathrm{~J}_{\mathrm{HH}}=5.8 \mathrm{~Hz}, 2 \mathrm{H}, \mathrm{C}(3)-\mathrm{Hpy}\right), 8.38$ $\left(\mathrm{d},{ }^{3} \mathrm{~J}_{\mathrm{HH}}=5.8 \mathrm{~Hz}, 2 \mathrm{H}, \mathrm{C}(2)-\mathrm{HPy}\right) ;{ }^{13} \mathrm{C} \mathrm{NMR}\left(75 \mathrm{MHz}, \mathrm{CD}_{2} \mathrm{Cl}_{2}\right.$, $\left.25{ }^{\circ} \mathrm{C}\right): \delta=18.2\left(o-\mathrm{CH}_{3}{ }^{1}\right), 19.1\left(o-\mathrm{CH}_{3}{ }^{2}\right), 21.1\left(\beta-\mathrm{CCH}_{3}\right), 23.4(p-$

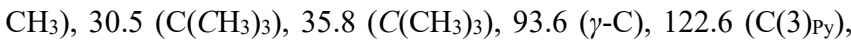
$128.9\left(m-\mathrm{C}^{1}\right), 129.5\left(m-\mathrm{C}^{2}\right), 131.6\left(o-\mathrm{C}^{1}\right), 133.0\left(o-\mathrm{C}^{2}\right), 134.0(p-$ C), $143.0(i-\mathrm{C}), 149.3$ (C(2)Рy), $165.3(\mathrm{C}(4) \mathrm{Py}), 168.3\left(\beta-\mathrm{CCH}_{3}\right)$.

dmap-Zn(Me) $\mathbf{L}^{2}$ (8): $\mathrm{L}^{2} \mathrm{ZnMe}(0.50 \mathrm{~g}, 1.0 \mathrm{mmol})$ and dmap (0.12 g, $1.0 \mathrm{mmol}$ ) were dissolved in $5 \mathrm{ml}$ of $\mathrm{CH}_{2} \mathrm{Cl}_{2}$ and the resulting mixture was stirred for $18 \mathrm{~h}$ at room temperature. The solution was concentrated to $2 \mathrm{~mL}$ and stored at $-30{ }^{\circ} \mathrm{C}$. Colorless crystals of $\mathbf{8}$ were formed within $24 \mathrm{~h}(0.56 \mathrm{~g}, 90 \%)$. Elemental analysis calcd (\%) for $\mathrm{C}_{37} \mathrm{H}_{54} \mathrm{~N}_{4} \mathrm{Zn}^{*} \mathrm{CH}_{2} \mathrm{Cl}_{2}$ : C 64.7, $\mathrm{H}$ 8.0, N 7.9; found: C 65.1, $\mathrm{H} 7.8, \mathrm{~N}$ 8.3. ${ }^{1} \mathrm{H}$ NMR $\left(300 \mathrm{MHz}, \mathrm{CD}_{2} \mathrm{Cl}_{2}, 20{ }^{\circ} \mathrm{C}\right): \delta=-1.24(\mathrm{~s}, 3 \mathrm{H}$, $\left.\mathrm{ZnCH}_{3}\right), 1.12\left(\mathrm{~d},{ }^{3} \mathrm{~J}_{\mathrm{HH}}=6.9 \mathrm{~Hz}, 12 \mathrm{H}, \mathrm{CH}\left(\mathrm{CH}_{3}{ }^{1}\right)_{2}\right), 1.26\left(\mathrm{~d},{ }^{3} \mathrm{~J}_{\mathrm{HH}}=\right.$ $\left.6.9 \mathrm{~Hz}, 12 \mathrm{H}, \mathrm{CH}\left(\mathrm{CH}_{3}{ }^{2}\right)_{2}\right), 1.79\left(\mathrm{~s}, 6 \mathrm{H}, \beta-\mathrm{CCH}_{3}\right), 3.01(\mathrm{~s}, 6 \mathrm{H}$, $\left.\mathrm{N}\left(\mathrm{CH}_{3}\right)_{2}\right), 3.07\left(\mathrm{sept},{ }^{3} \mathrm{~J}_{\mathrm{HH}}=6.9 \mathrm{~Hz}, 4 \mathrm{H}, \mathrm{CH}\left(\mathrm{CH}_{3}\right)_{2}\right), 5.07(\mathrm{~s}, 1 \mathrm{H}, \gamma-$ $\mathrm{CH}), 6.53\left(\mathrm{~d},{ }^{3} \mathrm{~J}_{\mathrm{HH}}=6.50 \mathrm{~Hz}, 2 \mathrm{H}, \mathrm{C}(3)-\mathrm{H}_{\mathrm{dmap}}\right), 7.19(\mathrm{~s}, 6 \mathrm{H}, m / p-\mathrm{H})$, $8.20\left(\mathrm{~d},{ }^{3} \mathrm{~J}_{\mathrm{HH}}=6.50 \mathrm{~Hz}, 2 \mathrm{H}, \mathrm{C}(2)-\mathrm{H}_{\mathrm{dmap}}\right) .{ }^{13} \mathrm{C} \mathrm{NMR}(75 \mathrm{MHz}$, $\left.\mathrm{CD}_{2} \mathrm{Cl}_{2}, 25{ }^{\circ} \mathrm{C}\right): \delta=-18.5\left(\mathrm{ZnCH}_{3}\right), 23.3\left(\mathrm{CH}\left(\mathrm{CH}_{3}{ }^{1}\right)_{2}\right), 23.4(\beta-$ $\left.\mathrm{CCH}_{3}\right), 24.0\left(\mathrm{CH}\left(\mathrm{CH}_{3}{ }^{2}\right)_{2}\right), 27.9\left(\mathrm{CH}\left(\mathrm{CH}_{3}\right)_{2}\right), 38.9\left(\mathrm{~N}\left(\mathrm{CH}_{3}\right)_{2}\right), 94.1$ $(\gamma-\mathrm{C}), 106.4\left(\mathrm{C}(3)_{\mathrm{dmap}}\right), 123.2(m-\mathrm{C}), 124.7(p-\mathrm{C}), 141.9(o-\mathrm{C})$, $145.3(i-\mathrm{C}), 149.5\left(\mathrm{C}(2)_{\mathrm{dmap}}\right), 154.5\left(\mathrm{C}(4)_{\mathrm{dmap}}\right), 166.8\left(\beta-\mathrm{CCH}_{3}\right)$.

$\boldsymbol{t}$-BuPy-Zn(Me)L $\mathbf{L}^{2}$ (9): $\mathrm{L}^{2} \mathrm{ZnMe}(0.50 \mathrm{~g}, 1.0 \mathrm{mmol})$ and t-BuPy $(0.14 \mathrm{~g}, 1.0 \mathrm{mmol})$ were dissolved in $5 \mathrm{ml}$ of $\mathrm{CH}_{2} \mathrm{Cl}_{2}$ and the resulting mixture was stirred for $18 \mathrm{~h}$ at room temperature. The solution was concentrated to $2 \mathrm{~mL}$ and stored at $-30{ }^{\circ} \mathrm{C}$. Colorless crystals of 9 were formed within $24 \mathrm{~h}(0.58 \mathrm{~g}, 91 \%)$. Elemental analysis calcd (\%) for $\mathrm{C}_{39} \mathrm{H}_{57} \mathrm{~N}_{3} \mathrm{Zn}^{*} \mathrm{C}_{2} \mathrm{H}_{4} \mathrm{Cl}_{4}$ : C 61.3, H 7.7, $\mathrm{N}$ 5.2; found: C 60.7, $\mathrm{H} \mathrm{8.3,} \mathrm{N} \mathrm{4.5.}{ }^{1} \mathrm{H}$ NMR (300 $\left.\mathrm{MHz}, \mathrm{CD}_{2} \mathrm{Cl}_{2}, 20^{\circ} \mathrm{C}\right)$ : $\delta=-1.23\left(\mathrm{~s}, 3 \mathrm{H}, \mathrm{ZnCH}_{3}\right), 1.12\left(\mathrm{~d},{ }^{3} \mathrm{~J}_{\mathrm{HH}}=6.8 \mathrm{~Hz}, 12 \mathrm{H}, \mathrm{CH}\left(\mathrm{CH}_{3}{ }^{1}\right)_{2}\right)$, $1.26\left(\mathrm{~d},{ }^{3} \mathrm{~J}_{\mathrm{HH}}=6.8 \mathrm{~Hz}, 12 \mathrm{H}, \mathrm{CH}\left(\mathrm{CH}_{3}{ }^{2}\right)_{2}\right), 1.35\left(\mathrm{~s}, 9 \mathrm{H}, \mathrm{C}\left(\mathrm{CH}_{3}\right)_{3}\right)$, $1.79\left(\mathrm{~s}, 6 \mathrm{H}, \beta-\mathrm{CCH}_{3}\right), 3.06$ (sept, $\left.{ }^{3} \mathrm{~J}_{\mathrm{HH}}=6.8 \mathrm{~Hz}, 4 \mathrm{H}, \mathrm{CH}\left(\mathrm{CH}_{3}\right)_{2}\right)$, $5.08(\mathrm{~s}, 1 \mathrm{H}, \gamma-\mathrm{CH}), 7.19(\mathrm{~s}, 6 \mathrm{H}, m / p-\mathrm{H}), 7.32\left(\mathrm{~d},{ }^{3} \mathrm{~J}_{\mathrm{HH}}=6.40 \mathrm{~Hz}\right.$, $2 \mathrm{H}, \mathrm{C}(3)-\mathrm{HPy}), 8.51\left(\mathrm{~s},{ }^{3} \mathrm{~J}_{\mathrm{HH}}=6.40 \mathrm{~Hz}, 2 \mathrm{H}, \mathrm{C}(2)-\mathrm{HPy}\right) .{ }^{13} \mathrm{C} \mathrm{NMR}$ $\left(75 \mathrm{MHz}, \mathrm{CD}_{2} \mathrm{Cl}_{2}, 25{ }^{\circ} \mathrm{C}\right): \delta=-17.6\left(\mathrm{ZnCH}_{3}\right), 23.0\left(\mathrm{CH}\left(\mathrm{CH}_{3}{ }^{1}\right)_{2}\right)$, $23.2 \quad\left(\beta-\mathrm{CCH}_{3}\right), \quad 23.9 \quad\left(\mathrm{CH}\left(\mathrm{CH}_{3}{ }^{2}\right)_{2}\right), \quad 27.9 \quad\left(\mathrm{CH}\left(\mathrm{CH}_{3}\right)_{2}\right), \quad 30.2$ $\left(\mathrm{C}\left(\mathrm{CH}_{3}\right)_{3}\right), 34.5\left(\mathrm{C}\left(\mathrm{CH}_{3}\right)_{3}\right), 94.8(\gamma-\mathrm{C}), 122.6\left(\mathrm{C}(3)_{\mathrm{Py}}\right), 123.3(m-\mathrm{C})$, 125.1 (p-C), $141.6(o-\mathrm{C}), 144.5$ (i-C), $149.3(\mathrm{C}(2)$ Рy $), 165.3$ (C(4)Рy), $167.3\left(\beta-\mathrm{CCH}_{3}\right)$.
Polymerization studies. A mixture of freshly prepared 1 ( $0.0683 \mathrm{~g}$, $0.05 \mathrm{mmol})$ and lactide $(1.4413 \mathrm{~g}, 10 \mathrm{mmol})$ was heated in the absence of any solvent over the melting point of lactide to $160{ }^{\circ} \mathrm{C}$ for $8 \mathrm{~min}$ in a glass reactor. After cooling to room temperature the less remaining monomer lactide on the reactor wall was dissolved in $\mathrm{CH}_{2} \mathrm{Cl}_{2}$ and separated. The crude polymer was slowly dissolved in $10 \mathrm{~mL}$ of THF, exposed to air and $10 \mathrm{~mL}$ of cold methanol was added. This solution was quenched in $80 \mathrm{~mL}$ of $n$-hexane and the white polymer precipitation was isolated by filtration and dried in vacuo.

Single crystal $\mathrm{X}$-ray analyses. X-ray crystal structures were collected using a Bruker AXS D8 Kappa diffractometer with APEX2 detector (MoKa radiation, $\lambda=0.71073 \AA$ ) at $T=100(1) \mathrm{K}$ (6 180(1)K). The structures were solved by Direct Methods (SHELXS-97) ${ }^{[33]}$ and refined by full-matrix least-squares on $\mathrm{F}^{2}$. Semi-empirical absorption corrections from equivalent reflections on basis of multi-scans (Bruker AXS APEX2) were applied. All non-hydrogen atoms were refined anisotropically and hydrogen atoms by a riding model. ${ }^{[34]}$ 2: $\left[\mathrm{C}_{37} \mathrm{H}_{49} \mathrm{~N}_{6} \mathrm{Zn}\right]\left[\mathrm{AlC}_{16} \mathrm{~F}_{36} \mathrm{O}_{4}\right], M=$ 1610.33 , colorless crystal $(0.32 \times 0.26 \times 0.18 \mathrm{~mm})$; monoclinic, space group $P 2{ }_{1} / n ; a=13.7265(3), b=23.7765(5), c=20.4753(5)$ $\AA ; \beta=107.2980(10)^{\circ}, V=6380.2(2) \AA^{3} ; Z=4 ; \mu=0.554 \mathrm{~mm}^{-1}$; $\rho_{\text {ber. }}=1.676 \mathrm{~g} \mathrm{~cm}^{-3} ; 89530$ reflexes $\left(2 \theta_{\max }=57^{\circ}\right), 16119$ unique $\left(R_{\text {int }}=0.0326\right)$; Goof $1.020 ; 1018$ parameters; largest max. $/ \mathrm{min}$. in the final difference Fourier synthesis $1.282 \mathrm{e} \AA^{-3} /-0.682 \mathrm{e} \AA^{-3}$; max./min. transmission $0.75 / 0.67 ; R_{1}=0.044(I>2 \sigma(I)), w R_{2}$ (all data $)=0.1237$. Perfluoromethyl groups (C92 to C94) are disorder over two sites with occupancies of 0.7 and 0.3 respectivly. The highest peaks of the residual electron density indicate further disorder of this group which could not be resolved. 3: $\left[\mathrm{C}_{47} \mathrm{H}_{67} \mathrm{~N}_{4} \mathrm{Zn}\right]\left[\mathrm{AlC}_{16} \mathrm{~F}_{36} \mathrm{O}_{4}\right] * 0.1 \mathrm{CH}_{2} \mathrm{Cl}_{2}, \quad M=1727.56$, colorless crystal $(0.30 \times 0.25 \times 0.07 \mathrm{~mm})$; orthorhombic, space group $P b c a$; $a=23.2213(7), b=20.5324(6), c=31.0581(9) \AA ; V=14808.2(8)$ $\AA^{3} ; Z=8 ; \mu=0.488 \mathrm{~mm}^{-1} ; \rho_{\text {ber. }}=1.550 \mathrm{~g} \mathrm{~cm}^{-3} ; 111316$ reflexes $\left(2 \theta_{\max }=50^{\circ},>43^{\circ}\right.$ approx. $65 \%$ unobserved, i.e. $\left.I<2 \sigma(I)\right)$, unique $\left(R_{\text {int }}=0.0651\right)$; Goof $1.052 ; 1001$ parameters; largest max. $/ \mathrm{min}$. in the final difference Fourier synthesis $0.860 \mathrm{e} \AA^{-3} /-0.811 \mathrm{e}^{-3}$; max./min. transmission $0.75 / 0.60 ; R_{1}=0.0508(I>2 \sigma(I)), w R_{2}$ (all data) $=0.1407$. The perfluoromethyl groups connected to $\mathrm{C} 300$ are disordered over at least two sites. Two of them could be identified but only refined isotropically with various restraints. The position of the residual electron density peaks suggests further sites which could not be modeled. The structure contains a dichloromethane whose site is partially occupied (about $10 \%$ ) which could also only be refined isotropically. Due to the poor crystal quality only the connectivity of the structure model should be regarded reliable. 4: $\left[\mathrm{C}_{43} \mathrm{H}_{61} \mathrm{~N}_{6} \mathrm{Zn}\right]\left[\mathrm{C}_{24} \mathrm{BF}_{20}\right] * \mathrm{CH}_{2} \mathrm{Cl}_{2}, M=1491.32$, colorless crystal $(0.33 \times 0.18 \times 0.13 \mathrm{~mm})$; triclinic, space group $P-1 ; a=$ 12.7348(3), $b=16.5546(4), c=17.9992(4) \AA ; \alpha=65.7970(10)^{\circ}, \beta$ $=75.9020(10)^{\circ}, \gamma=84.9610(10)^{\circ}, V=3356.48(14) \AA^{3} ; Z=2 ; \mu=$ $0.548 \mathrm{~mm}^{-1} ; \rho_{\text {ber. }}=1.476 \mathrm{~g} \mathrm{~cm}^{-3} ; 66800$ reflexes $\left(2 \theta_{\max }=56^{\circ}\right)$, 15951 unique $\left(R_{\text {int }}=0.0228\right)$; Goof $1.043 ; 883$ parameters; largest max./min. in the final difference Fourier synthesis $1.097 \mathrm{e} \AA^{-3} /-$ $1.566 \mathrm{e}^{-3}$; max./min. transmission $0.75 / 0.68 ; R_{1}=0.0483(I>$ $2 \sigma(I)), w R_{2}$ (all data) $=0.1376 .5:\left[\mathrm{C}_{47} \mathrm{H}_{67} \mathrm{~N}_{4} \mathrm{Zn}\right]\left[\mathrm{C}_{24} \mathrm{BF}_{20}\right]^{*} \mathrm{CH}_{2} \mathrm{Cl}_{2}$, $M=1517.39$, colorless crystal $(0.38 \times 0.33 \times 0.25 \mathrm{~mm})$; monclinic, space group $P 2{ }_{1} / c ; a=13.2455(2), b=25.5145(5), c=22.0286(4)$ $\AA ; \beta=105.598(1)^{\circ}, V=7170.4(2) \AA^{3} ; Z=4 ; \mu=0.513 \mathrm{~mm}^{-1} ; \rho_{b e r}$. $=1.406 \mathrm{~g} \mathrm{~cm}^{-3} ; 108166$ reflexes $\left(2 \theta_{\max }=58^{\circ}\right), 19351$ unique $\left(R_{\text {int }}\right.$ $=0.0258)$; Goof 1.087; 901 parameters; largest max./min. in the final difference Fourier synthesis $1.905 \mathrm{e}^{-3}(1.05 \AA$ from CL2)/$1.082 \mathrm{e}^{-3}$; max./min. transmission $0.75 / 0.65 ; R_{1}=0.0445(I>$ $2 \sigma(I)), w R_{2}($ all data $)=0.1290$. The refinement was performed with 
solvent-free reflection data following PLATON/squeeze run. A refinement of the untreated reflection data set produces several peaks with $2.5-7.5 \mathrm{e} / \AA^{3}$ corresponding to highly disordered dichloromethane molecule. The peaks attributed to two chlorine and one carbon atoms over two sites with occupancies factor 0.5 result in $\mathrm{R} 1=0.0783$. The SQUEEZE refinement revealed 4 voids corresponding to $119 \AA^{3}$ each. 6: $\mathrm{C}_{24} \mathrm{H}_{32} \mathrm{~N}_{2} \mathrm{Zn}, M=413.89$, colorless crystal $(0.38 \times 0.32 \times 0.23 \mathrm{~mm})$; monoclinic, space group $P 21 / n ; a=8.8277(10), b=32.260(4), c=16.7973(18) \AA ; \beta=$ $104.955(4)^{\circ}, V=4621.6(9) \quad \AA^{3} ; Z=8 ; \mu=1.072 \mathrm{~mm}^{-1} ; \rho_{\text {ber. }}=$ $1.190 \mathrm{~g} \mathrm{~cm}^{-3} ; 71621$ reflexes $\left(2 \theta_{\max }=55^{\circ}\right), 11467$ unique $\left(R_{\text {int }}=\right.$ $0.0313)$; Goof 1.099; 487 parameters; largest max./min. in the final difference Fourier synthesis $0.381 \mathrm{e} \AA^{-3} /-0.541 \mathrm{e} \AA^{-3}$; max. $/ \mathrm{min}$. transmission $0.75 / 0.52 ; R_{1}=0.0400(I>2 \sigma(I)), w R_{2}($ all data $)=$ 0.0969. 7: $\mathrm{C}_{32} \mathrm{H}_{42} \mathrm{ClN}_{3} \mathrm{Zn}, M=569.51$, colorless crystal $(0.38 \mathrm{x}$ $0.12 \times 0.08 \mathrm{~mm})$; triclinic, space group $P-1 ; a=7.9599(2), b=$ 12.2162(3), $c=16.4476(4) \AA ; \alpha=109.169$ (1), $\beta=93.783$ (1), $\gamma=$ 92.229 (1), $V=1504.28(6) \AA^{3} ; Z=2 ; \mu=0.929 \mathrm{~mm}^{-1} ; \rho_{\text {ber. }}=$ $1.257 \mathrm{~g} \mathrm{~cm}^{-3} ; 43585$ reflexes $\left(2 \theta_{\max }=58^{\circ}\right), 7808$ unique $\left(R_{\text {int }}=\right.$ $0.0284)$; Goof 1.036 ; 334 parameters; largest max. $/ \mathrm{min}$. in the final difference Fourier synthesis $0.430 \mathrm{e} \AA^{-3} /-0.316 \mathrm{e} \AA^{-3}$; max. $/ \mathrm{min}$. transmission $0.75 / 0.65 ; R_{1}=0.0282(I>2 \sigma(I)), w R_{2}($ all data $)=$ 0.0727. 8: $\mathrm{C}_{37} \mathrm{H}_{54} \mathrm{~N}_{4} \mathrm{Zn} * 3\left(\mathrm{CH}_{2} \mathrm{Cl}_{2}\right), M=$, colorless crystal $(0.37 \mathrm{x}$ $0.12 \times 0.10 \mathrm{~mm})$; triclinic, space group $P-1 ; a=9.2948(3), b=$ 12.2388(4), $c=20.7088(7) \AA ; \alpha=104.079(2) \beta=102.4820(10), \gamma$ $=95.013(2), V=2206.50(13) \AA^{3} ; Z=2 ; \mu=0.952 \mathrm{~mm}^{-1}$; $\rho$ ber. $=$ $1.317 \mathrm{~g} \mathrm{~cm}^{-3} ; 42837$ reflexes $\left(2 \theta_{\max }=58^{\circ}\right), 10961$ unique $\left(R_{\text {int }}=\right.$ 0.0332 ); Goof $1.050 ; 460$ parameters; largest max. $/ \mathrm{min}$. in the final difference Fourier synthesis $1.675 \mathrm{e} \AA^{-3} /-1.260 \mathrm{e} \AA^{-3}$; max./min. transmission $0.75 / 0.57 ; R_{1}=0.0538(I>2 \sigma(I)), w R_{2}($ all data $)=$ 0.1494. 9: $\mathrm{C}_{3}{ }_{9} \mathrm{H}_{57} \mathrm{~N}_{3} \mathrm{Zn} * \mathrm{CH}_{2} \mathrm{Cl}_{2}, M=718.17$, colorless crystal $(0.37 \times 0.32 \times 0.23 \mathrm{~mm})$; monoclinic, space group $P 21 / n ; a=$ 16.7558(10), $b=13.5456(8), c=17.8375(10) \AA ; \beta=101.587(2)^{\circ}$, $V=3966.0(4) \AA^{3} ; Z=4 ; \mu=0.784 \mathrm{~mm}^{-1} ; \rho_{\text {ber. }}=1.203 \mathrm{~g} \mathrm{~cm}^{-3}$; 50562 reflexes $\left(2 \theta_{\max }=60^{\circ}\right), 11425$ unique $\left(R_{\text {int }}=0.0234\right)$; Goof $1.034 ; 415$ parameters; largest max./min. in the final difference Fourier synthesis $0.885 \mathrm{e}^{-3} /-0.736 \mathrm{e} \AA^{-3}$; max./min. transmission $0.75 / 0.67 ; R_{1}=0.0326(I>2 \sigma(I)), w R_{2}($ all data $)=0.0928$.

Supporting Information: X-ray crystallographic data of $\mathbf{2}$ - 9 are given in the electronic supplement. The crystallographic data (without structure factors) were also deposited as ,supplementary publication no. CCDC 91429 (2), 914735 (3), 914731 (4), 914734 (5), 914736 (6) 914730 (7), 914733 (8), 914732 (9) at the Cambridge Crystallographic Data Centre. These data can be obtained free of charge from The Cambridge Crystallographic Data Centre: CCDC, 12 Union Road, Cambridge, CB21EZ (Fax: (+44)1223/336033; E-mail: deposit@ccdc.cam-ak.uk).

\section{Acknowledgments}

S. Schulz likes to thank the University of Duisburg-Essen for financial support.

[1] a) B. J. O'Keefe, M. A. Hillmyer, W. B. Tolman, J. Chem. Soc., Dalton Trans. 2001, 15, 2215-2224. b) M. J. Stanford, A. P. Dove, Chem. Soc. Rev. 2010, 39, 486-494. c) O. Dechy-Cabaret, B. Martin-Vaca, D. Bourissou, Chem. Rev. 2004, 104, 6147-6176. d) C. M. Thomas, Chem. Soc. Rev. 2010, 39, 165-173.

[2] H. R. Kricheldorf, Chem. Rev. 2009, 109, 5579-5594.

[3] a) A. P. Gupta, V. Kumar, Eur. Polym. J. 2007, 43, 40534404. b) D. Garlotta, J. Polymer. Environ. 2002, 9, 63-84.
[4] a) O. Dechy-Cabaret, B. Martin-Vaca, D. Bourissou, Chem. Rev. 2004, 104, 6147-6176. b) G. Schwach, J. Coudane, R. Engel, M. Vert, Polym. Chem. 1997, 35, 3431-3440.

[5] a) J. Börner, I. dos Santos Vieira, A. Pawlis, A. Döring, D. Kuckling, S. Herres-Pawlis, Chem. Eur. J. 2011, 17, 45074512. b) C. M. Silvernail, L. J. Yao, L. M. R. Hill, M. A. Hillmyer, W. B. Tolman, Inorg. Chem. 2007, 46, 6565-6574. c) D. J. Darensbourg, O. Karroonnirun, Inorg. Chem. 2010, 49, 2360-2371. d) M. Cheng, A. B. Attygalle, E. B. Lobkovsky, G. W. Coates, J. Am. Chem. Soc. 1999, 121, 11583-11584. e) S. Song, X. Zhang, H. Ma, Y. Yang, Dalton Trans. 2012, 41, 3266-3277. f) I. D'Auria, M. Lamberti, M. Mazzeo, S. Milione, G. Roviello, C. Pellecchia, Chem. Eur. J. 2012, 18, 2349-2360.

[6] a) K. Matsubara, C. Terata, H. Sekine, K. Yamatani, T. Harada, K. Eda, M. Dan, Y. Koga, M. Yasuniwa, J. Polym. Sci., Part A: Polym. Chem. 2012, 50, 957-966. b) H.-L. Chen, S. Dutta, P.-Y. Huang, C.-C. Lin, Organometallics 2012, 31, 2016-2025. c) J. Weil, R. T. Mathers, Y. D. Y. L. Getzler, Macromolecules 2012, 45, 1118-1121. d) T. M. Ovitt, G. W. Coates, J. Am. Chem. Soc. 2002, 124, 1316-1326. e) Y. Wang, H. Ma, Chem. Commun. 2012, 48, 6729-6731. f) W. Zhao, Y. Wang, X. Liu, X. Chen, D. Cui, E. Y.-X. Chen, Chem. Commun. 2012, 48, 6375-6377.

[7] a) Y. Wang, W. Zhao, D. Liu, S. Li, X. Liu, D. Cui, X. Chen, Organometallics 2012, 31, 4182-4190. b) Y. Sarazin, M. Schormann, M. Bochmann, Organometallics 2004, 23, 32963302. c) H.-Y. Chen, L. Mialon, K. A. Abboud, S. A. Miller, Organometallics 2012, 31, 5252-5261.

[8] a) R. H. Platel, A. J. P. White, C. K. Williams, Inorg. Chem. 2011, 50, 7718-7728. b) M. Bouyahyi, N. Ajellal, E. Kirillov, C. M. Thomas, J.-F. Carpentier, Chem. Eur. J. 2011, 17, 1872-1883. c) T.-P.-A. Cao, A. Buchard, X. F. Le Goff, A. Auffrant, C. K. Williams, Inorg. Chem. 2012, 51, 2157-2169.

[9] W. Dittrich, R. Schulz, Angew. Makromol. Chem. 1971, 15, 109-126.

[10] H. R. Kricheldorf, M. Berl, N. Scharnagl, Marcromol. 1988, 21, 286-293.

[11] a) B. M. Chamberlain, M. Cheng, D. R. Moore, T. M. Ovitt, E. B. Lobkovsky, G. W. Coates, J. Am. Chem. Soc. 2001, 123, 3229-3238; b) O. Dechy-Cabaret, B. Martin-Vaca, D. Bourissou, Chem. Rev. 2004, 104, 6147-6176.

[12] G. W. Coates, D. R. Moore, Angew. Chem. 2004, 116, 6784 6806; Angew. Chem. Int. Ed. 2004, 43, 6618-6639; b) D. J. Darensbourg, Chem. Rev. 2007, 107, 2388-2410; c) M. R. Kember, A. Buchard, C. K. Williams, Chem. Commun. 2011, 47, 141-163; d) S. Klaus, M. W. Lehenmeier, C. E. Anderson, B. Rieger, Coord. Chem. Rev. 2011, 255, 14601479.

[13] W. Kaminsky, A. Funck, H. Hähnsen, Dalton Trans. 2009, 41, 9052-9057.

[14] C. A. Wheaton, P. G. Hayes, B. J. Ireland, Dalton Trans. 2009, 4832-4846.

[15] (a) M. Reinmuth, U. Wild, D. Rudolf, E. Kaifer, M. Enders, H. Wadepohl, H.-J. Himmel, Eur. J. Inorg. Chem. 2009, 4795-4808; (b) M. D. Hannant, M. Schormann, M. Bochmann, Dalton Trans. 2002, 4071-4073; (c) S. Dagorne, M. Bouyahyi, J. Vergnaud, J.-F. Carpentier, Organometallics 2010, 29, 1865-1868; (d) S. Dagorne, S. Bellemin-Laponnaz, R. Welter, Organometallics 2004, 23, 3053-3061; (e) H. E. Dyer, S. Huijser, A. D. Schwarz, C. Wang, R. Duchateau P. Mountford, Dalton Trans. 2008, 32-35; (f) M. D. Hannant, M. Schormann, D. L. Hughes, M. Bochmann, Inorg. Chim. Acta 2005, 358, 1683-1691.

[16] (a) Y. Sarazin, B. Liu, T. Roisnel, L. Maron, J.-F. Carpentier, J. Am. Chem. Soc. 2011, 133, 9069-9087; (b) M. G. Cushion, P. Mountford, Chem. Commun. 2011, 47, 2276-2278; (c) Y. Sarazin, V. Poirier, T. Roisnel, J.-F. Carpentier, Eur. J. Inorg. Chem. 2010, 3423-3428; (d) J. Boerner, S. Herres-Pawlis, U. Flörke, K. Huber, Eur. J. Inorg. Chem. 2007, 5645-5651; (e) 
S. Dagorne, F. Le Bideau, R. Welter, S. Bellemin-Laponnaz, A. Maisse-Francois, Chem. Eur. J. 2007, 13, 3202-3217; (f) E. Piedra-Arroni, P. Brignou, A. Amgoune, S. M. Guillaume, J.-F. Carpentier, D. Bourissou, Chem. Commun. 2011, 47, 9828-9830; (g) P. Brignou, S. M. Guillaume, T. Roisnel, D. Bourissou, J.-F. Carpentier, Chem. Eur. J. 2012, 18, 93609370; (h) B. Liu, V. Dorcet, L. Maron, J.-F. Carpentier, Y. Sarazin, Eur. J. Inorg. Chem. 2012, 18, 3023-3031; (i) B. Liu, T. Roisnel, Y. Sarazin, Inorg. Chim. Acta 2012, 380, 2 13; j) T. R. Jensen, C. P. Schaller, M. A. Hillmyer, W. B. Tolman, J. Organomet. Chem. 2005, 690, 5881-5891; k) J. Börner, U. Flörke, K. Huber, A. Döring, D. Kuckling, S. Herres-Pawlis, Chem. Eur. J. 2009, 15, 2362-2376; 1) B. J. Ireland, C. A. Wheaton, P. G. Hayes, Organometallics 2010, 29, 1079-1084; m) J. Börner, U. Flörke, T. Glöge, T. Bannenberg, M. Tamm, M. D. Jones, A. Döring, D. Kuckling, S. Herres-Pawlis, J. Mol. Catal. A 2010, 316, 139145.

[17] a) M. M. Ibrahim, C. P. Olmo, T. Tekeste, J. Seebacher, G. He, J. A. M. Calvo, K. Böhmerle, G. Steinfeld, H. Brombacher, H. Vahrenkamp, Inorg. Chem. 2006, 45, 74937502 ; b) L. E. Garner, H. Zhu, M. L. Hlavinka, J. R. Hagadorn, E. Y.-X. Chen, J. Am. Chem. Soc. 2006, 128, $14822-14823$.

[18] C. A. Wheaton, B. J. Ireland, P. G. Hayes, Organometallics 2009, 28, 1282-1285.

[19] C. A. Wheaton, P. G. Hayes, Dalton Trans. 2010, 39, 38613869.

[20] C. A. Wheaton, P. G. Hayes, Chem. Commun. 2010, 46, 8404-8406.

[21] C. A. Wheaton, P. G. Hayes, Catal. Sci. Technol. 2012, 2, $125-138$.

[22] C. A. Wheaton, P. G. Hayes, J. Organomet. Chem. 2012, 704, 65-69.

[23] C. Romain, V. Rosa, C. Fliedel, F. Bier, F. Hild, R. Welter, S. Dagorne, T. Avilés, Dalton Trans. 2012, 41, 3377-3379.

[24] J. Prust, A. Stasch, W. Zheng, H. W. Roesky, E. Alexopoulos, I. Usón, D. Böhler, T. Schuchardt, Organometallics 2001, 20, 3825-3828.

[25] I. Krossing, Chem. Eur. J. 2001, 2, 490-502.

[26] I. Krossing, I. Raabe, Angew. Chem. 2004, 116, 2116-2142; Angew. Chem. Int. Ed. 2004, 43, 2066-2090.

[27] S. Schmidt, S. Schulz, D. Bläser, R. Boese, M. Bolte, Organometallics 2010, 29, 6097-6103.

[28] a) H.-Y. Chen, H.-Y. Tang, C.-C. Lin, Macromolecules 2006, 39, 3745-3752. b) R. J. Wehmschulte, L. Wojtas, Inorg. Chem. 2011, 50, 11300-11302. c) C. Lichtenberg, T. P.
Spaniol, J. Okuda, Angew. Chem. 2012, 124, 8225-8229; Angew. Chem. Int. Ed. 2012, 51, 8101-8105.

[29] a) S. Schulz, T. Eisenmann, D. Schuchmann, M. Bolte, M. Kirchner, R. Boese, J. Spielmann, S. Harder, Z. Naturforsch 2009, 64b, 1397-1400; b) S. Schulz, T. Eisenmann, D. Bläser, R. Boese, Z. Anorg. Allg. Chem. 2009, 635, 995-1000; c) S. Schulz, T. Eisenmann, U. Westphal, S. Schmidt, U. Flörke, $Z$. Anorg. Allg. Chem. 2009, 635, 216-220; d) S. Schulz, T. Eisenmann, S. Schmidt, D. Bläser, U. Westphal, R. Boese, Chem. Commun. 2010, 46, 7226-7228; e) G. Bendt, S. Schulz, J. Spielmann, S. Schmidt, D. Bläser, Christoph Wölper, Eur. J. Inorg. Chem. 2012, 3725-3731; f) M. H. Chisholm, J. C. Gallucci, K. Phomphrai, Inorg. Chem. 2005 44, 8004-8010; g) A. P. Dove, V. C. Gibson, E. L. Marshall, A. J. P. White, D. J. Williams, Dalton Trans. 2004, 570-578; h) L. R. Rieth, D. R. Moore, E. B. Lobkovsky and G. W. Coates, J. Am. Chem. Soc. 2002, 124, 15239-15248; i) M. H. Chisholm, J. C. Huffman, K. Phomphrai, Dalton Trans. 2001, 222-224; j) B. J. O'Keefe, M. A. Hillmeyer, W. B. Tolman, Dalton Trans. 2001, 2215-2224; k) M. Cheng, A. B. Attygalle, E. B. Lobkovsky, G. W. Coates, J. Am. Chem. Soc. 1999, 121, 11583-11584; 1) D. R. Moore, M. Cheng, E. B. Lobkovsky, G. W. Coates, J. Am. Chem. Soc. 2003, 125 , 11911-11924; m) M. Cheng, D. R. Moore, J. J. Reczek, B. M. Chamberlain, E. B. Lobkovsky, G. W. Coates, J. Am. Chem. Soc. 2001, 123, 8738-8749.

[30] M. Stender, R. J. Wright, B. E. Eichler, J. Prust, M. M. Olmstead, H. W. Roesky, P. P. Power, Dalton Trans. 2001, 23, 3465-3469.

[31] H. Brands, R. Feuerhake, S. Koenig, I. Krossing, J. Fluor Chem. 2001, 112, 83-90.

[32] J. B. Lambert, S. Zhang, S. M. Ciro, Organometallics 1994, 13, 2430-2443.

[33] G. M. Sheldrick, Acta Crystallogr. Sect. A 1990, 46, 467-473.

[34] a) G. M. Sheldrick, Acta Crystallogr. Sect. A 2008, A64, 112 122. b) G. M. Sheldrick, SHELXL-97, Program for Crystal Structure Refinement, Universität Göttingen 1997.

Received: ((will be filled in by the editorial staff)) Published online: ((will be filled in by the editorial staff)) 


\section{Entry for the Table of Contents}

\section{Layout 1:}

((Key Topic))

Several cationic zinc complex bearing weakly coordinating anions were synthesized and structurally characterized by single crystal X-ray diffraction. Preliminary results on the ROP of lactide using base-free cation 1 as catalyst are presented.

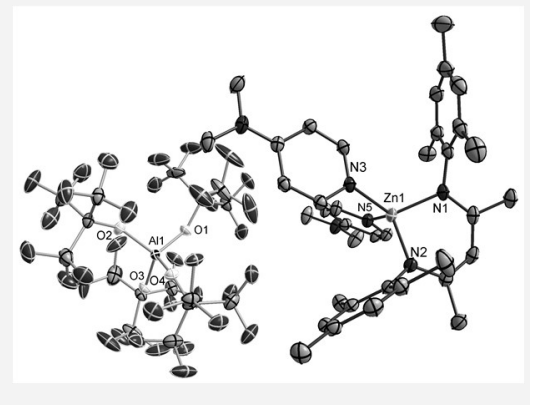

C. Scheiper, S. Schulz,* C. Wölper, D. Bläser, J. Roll ........ Page No. Page No.

Synthesis and Single Crystal X-ray Structures of Cationic Zinc $\beta$-Diketiminate Complexes

Keywords: Cations / Zinc / X-ray Analysis 

by referee 1)

Single crystal X-ray analyses. X-ray crystal structures were collected using a Bruker AXS D8 Kappa diffractometer with APEX2 detector (MoK $\alpha$ radiation, $\lambda=0.71073 \AA$ ). The structures were solved by Direct Methods (SHELXS-97) ${ }^{[33]}$ and refined by full-matrix least-squares on $\mathrm{F}^{2}$. Semi-empirical absorption corrections from equivalent reflections on basis of multi-scans (Bruker AXS APEX2) were applied. All non-hydrogen atoms were refined anisotropically and hydrogen atoms by a riding model. ${ }^{[34]}$

Table 1: Crystallographic details for complexes 2 - 9.

\begin{tabular}{|c|c|c|c|c|c|c|c|c|}
\hline & $2^{[\mathrm{a}]}$ & $3^{[\mathbf{b}]}$ & 4 & $5^{[\mathrm{c}]}$ & 6 & 7 & 8 & 9 \\
\hline $\begin{array}{l}\text { Empirical } \\
\text { formula }\end{array}$ & $\begin{array}{l}{\left[\mathrm{C}_{37} \mathrm{H}_{49} \mathrm{~N}_{6} \mathrm{Zn}\right]} \\
{\left[\mathrm{AlC}_{16} \mathrm{~F}_{36} \mathrm{O}_{4}\right]}\end{array}$ & $\begin{array}{l}{\left[\mathrm{C}_{47} \mathrm{H}_{67} \mathrm{~N}_{4} \mathrm{Zn}\right]} \\
{\left[\mathrm{AlC}_{16} \mathrm{~F}_{36} \mathrm{O}_{4}\right] \cdot} \\
0.1 \mathrm{CH}_{2} \mathrm{Cl}_{2}\end{array}$ & $\begin{array}{l}{\left[\mathrm{C}_{43} \mathrm{H}_{61} \mathrm{~N}_{6} \mathrm{Zn}\right]} \\
{\left[\mathrm{C}_{24} \mathrm{BF}_{20}\right] \cdot \mathrm{CH}_{2} \mathrm{Cl}_{2}}\end{array}$ & $\begin{array}{l}{\left[\mathrm{C}_{47} \mathrm{H}_{67} \mathrm{~N}_{4} \mathrm{Zn}\right]} \\
{\left[\mathrm{C}_{24} \mathrm{BF}_{20} \cdot \mathrm{CH}_{2} \mathrm{Cl}_{2}\right.}\end{array}$ & $\mathrm{C}_{24} \mathrm{H}_{32} \mathrm{~N}_{2} \mathrm{Zn}$ & $\mathrm{C}_{32} \mathrm{H}_{42} \mathrm{ClN}_{3} \mathrm{Zn}$ & $\begin{array}{l}\mathrm{C}_{37} \mathrm{H}_{54} \mathrm{~N}_{4} \mathrm{Zn} \cdot 3 \\
\mathrm{CH}_{2} \mathrm{Cl}_{2}\end{array}$ & $\begin{array}{l}\mathrm{C}_{39} \mathrm{H}_{57} \mathrm{~N}_{3} \mathrm{Zn} \cdot \\
\mathrm{CH}_{2} \mathrm{Cl}_{2}\end{array}$ \\
\hline Molecular mass & 1610.33 & 1727.56 & 1491.32 & 1517.39 & 413.89 & 569.51 & 874.99 & 718.17 \\
\hline Crystal system & monoclinic & orthorhombic & triclinic & monclinic & monoclinic & triclinic & triclinic & monoclinic \\
\hline Space group & $P 2_{1} / n$ & $P b c a$ & $P-1$ & $P 2_{1} / c$ & $P 2_{1} / n$ & $P-1$ & $P-1$ & $P 2_{1} / n$ \\
\hline $\mathrm{T}[\mathrm{K}]$ & $100(1)$ & $100(1)$ & $100(1)$ & $100(1)$ & $180(1)$ & $100(1)$ & $100(1)$ & $100(1)$ \\
\hline $\mathrm{a}[\AA]$ & $13.7265(3)$ & $23.2213(7)$ & $12.7348(3)$ & $13.2455(2)$ & $8.8277(10)$ & 7.9599(2) & $9.2948(3)$ & $16.7558(10)$ \\
\hline $\mathrm{b}[\AA]$ & $23.7765(5)$ & $20.5324(6)$ & $16.5546(4)$ & $25.5145(5)$ & $32.260(4)$ & $12.2162(3)$ & $12.2388(4)$ & $13.5456(8)$ \\
\hline c $[\AA]$ & $20.4753(5)$ & $31.0581(9)$ & $17.9992(4)$ & $22.0286(4)$ & $16.7973(18)$ & $16.4476(4)$ & $20.7088(7)$ & $17.8375(10)$ \\
\hline$\alpha\left[^{\circ}\right]$ & - & - & $65.7970(10)$ & - & - & $109.169(1)$ & $104.079(2)$ & - \\
\hline$\beta\left[^{\circ}\right]$ & $107.298(1)$ & - & $75.9020(10)$ & $105.598(1)$ & $104.955(4)$ & $93.783(1)$ & $102.482(1)$ & $101.587(2)$ \\
\hline$\gamma\left[{ }^{\circ}\right]$ & - & - & $84.9610(10)$ & - & - & $92.229(1)$ & $95.013(2)$ & - \\
\hline $\mathrm{V}\left[\AA^{3}\right]$ & $6380.2(2)$ & $14808.2(8)$ & $3356.48(14)$ & $7170.4(2)$ & $4621.6(9)$ & $1504.28(6)$ & $2206.50(13)$ & $3966.0(4)$ \\
\hline Z & 4 & 8 & 2 & 4 & 8 & 2 & 2 & 4 \\
\hline$\mu\left[\mathrm{mm}^{-1}\right]$ & 0.554 & 0.488 & 0.548 & 0.513 & 1.072 & 0.929 & 0.952 & 0.784 \\
\hline$D_{\text {calcd. }}\left[\mathrm{g} \mathrm{cm}^{-3}\right]$ & 1.676 & 1.550 & 1.476 & 1.406 & 1.190 & 1.257 & 1.317 & 1.203 \\
\hline $\begin{array}{l}\text { Crystal dim. } \\
{[\mathrm{mm}]}\end{array}$ & $\begin{array}{l}0.32 \times 0.26 \times \\
0.18\end{array}$ & $\begin{array}{l}0.30 \times 0.25 \times \\
0.07\end{array}$ & $0.33 \times 0.18 \times 0.13$ & $\begin{array}{l}0.38 \times 0.33 \times \\
0.25\end{array}$ & $\begin{array}{l}0.38 \times 0.32 \\
\times 0.23\end{array}$ & $\begin{array}{l}0.38 \times 0.12 \times \\
0.08\end{array}$ & $\begin{array}{l}0.37 \times 0.12 \times \\
0.10\end{array}$ & $\begin{array}{l}0.37 \times 0.32 \times \\
0.23\end{array}$ \\
\hline No. of reflections & 89530 & 111316 & 66800 & 108166 & 71621 & 43585 & 42837 & 50562 \\
\hline $\begin{array}{l}\text { No. of unique } \\
\text { reflections }\end{array}$ & 16119 & 12637 & 15951 & 19351 & 11467 & 7808 & 10961 & 11425 \\
\hline $\begin{array}{l}\max . / \min . \\
\text { transmission }\end{array}$ & $0.75 / 0.67$ & $0.75 / 0.60$ & $0.75 / 0.68$ & $0.75 / 0.65$ & $0.75 / 0.52$ & $0.75 / 0.65$ & $0.75 / 0.57$ & $0.75 / 0.67$ \\
\hline$R_{\text {merg }}$ & 0.0326 & 0.0651 & 0.0228 & 0.0258 & 0.0313 & 0.0284 & 0.0332 & 0.0234 \\
\hline $\begin{array}{l}\text { No. param. ref. / } \\
\text { restraints }\end{array}$ & 1018 / 0 & $1001 / 1059$ & $883 / 0$ & $901 / 0$ & $487 / 0$ & $334 / 0$ & $460 / 0$ & $415 / 0$ \\
\hline $\mathrm{R}_{1}$ & 0.044 & 0.0508 & 0.0483 & 0.0445 & 0.0400 & 0.0282 & 0.0538 & 0.0326 \\
\hline$w \mathrm{R}_{2}$ & 0.1237 & 0.1407 & 0.1376 & 0.1290 & 0.0969 & 0.0727 & 0.1494 & 0.0928 \\
\hline Goodness of fit ${ }^{[\mathrm{c}]}$ & 1.020 & 1.052 & 1.043 & 1.087 & 1.099 & 1.036 & 1.050 & 1.034 \\
\hline $\begin{array}{l}\text { Final max/min. } \\
\Delta \rho, \text { e } \AA^{-3}\end{array}$ & $1.282 /-0.682$ & $0.860 /-0.811$ & $1.097 /-1.566$ & $\begin{array}{l}1.905(1.05 \AA \\
\text { from Cl2)/-1.082 }\end{array}$ & $\begin{array}{l}0.381 /- \\
0.541\end{array}$ & $0.430 /-0.316$ & $1.675 /-1.260$ & $0.885 /-0.736$ \\
\hline
\end{tabular}

[a] Perfluoromethyl groups (C92 to C94) are disorder over two sites with occupancies of 0.7 and 0.3 respectively. The highest peaks of the residual electron density indicate further disorder of this group which could not be resolved.

[b] The perfluoromethyl groups connected to C300 are disordered over at least two sites. Two of them could be identified but only refined isotropically with various restraints. The position of the residual electron density peaks suggests further sites which could not be modeled. The structure contains a dichloromethane whose site is partially occupied (about $10 \%$ ) which could also only be refined isotropically. Due to the poor crystal quality only the connectivity of the structure model should be regarded reliable.

[c] The refinement was performed with solvent-free reflection data following PLATON/squeeze run. A refinement of the untreated reflection data set produces several peaks with $2.5-7.5 \mathrm{e} / \AA^{3}$ corresponding to highly disordered dichloromethane molecule. The peaks attributed to two chlorine and one carbon atoms over two sites with occupancies factor 0.5 result in R1 $=0.0783$. The SQUEEZE refinement revealed 4 voids corresponding to $119 \AA^{3}$ each. 
This text is made available via DuEPublico, the institutional repository of the University of Duisburg-Essen. This version may eventually differ from another version distributed by a commercial publisher.

DOI: $\quad 10.1002 /$ zaac. 201300119

URN: urn:nbn:de:hbz:464-20201116-103011-2

This is the peer reviewed version of the following article: Z. Anorg. Allg . Chem. 2013, 639, 216-220, which has been published in final form at:

https://doi.org/10.1002/zaac.201300119

All rights reserved. 